\title{
PARP-2 sustains erythropoiesis in mice by limiting replicative stress in erythroid progenitors
}

\author{
J Farrés ${ }^{1,9}$, L Llacuna ${ }^{1,9}$, J Martin-Caballero ${ }^{2}$, C Martínez ${ }^{3}$, JJ Lozano ${ }^{3}$, C Ampurdanés ${ }^{1}$, AJ López-Contreras ${ }^{4}$, L Florensa ${ }^{1,5}$, J Navarro ${ }^{1}$, \\ E Ottina ${ }^{6}$, F Dantzer $^{7}$, V Schreiber ${ }^{7}$, A Villunger ${ }^{6}, 0$ Fernández-Capetillo ${ }^{4}$ and J Yélamos ${ }^{*, 1,3,8}$
}

Erythropoiesis is a tightly regulated process in which multipotential hematopoietic stem cells produce mature red blood cells. Here we show that deletion of poly(ADP-ribose) polymerase-2 (PARP-2) in mice leads to chronic anemia at steady state, despite increased erythropoietin plasma levels, a phenomenon not observed in mice lacking PARP-1. Loss of PARP-2 causes shortened lifespan of erythrocytes and impaired differentiation of erythroid progenitors. In erythroblasts, PARP-2 deficiency triggers replicative stress, as indicated by the presence of micronuclei, the accumulation of $\gamma-\mathrm{H} 2 \mathrm{AX}$ (phospho-histone H2AX) in S-phase cells and constitutive CHK1 and replication protein A phosphorylation. Transcriptome analyses revealed the activation of the p53dependent DNA-damage response pathways in PARP-2-deficient cells, culminating in the upregulation of cell-cycle and cell death regulators, concomitant with G2/M arrest and apoptosis. Strikingly, while loss of the proapoptotic $\mathrm{p53}$ target gene Puma restored hematocrit levels in the PARP-2-deficient mice, loss of the cell-cycle regulator and CDK inhibitor p21 leads to perinatal death by exacerbating impaired fetal liver erythropoiesis in PARP-2-deficient embryos. Although the anemia displayed by PARP-2-deficient mice is compatible with life, mice die rapidly when exposed to stress-induced enhanced hemolysis. Our results pinpoint an essential role for PARP-2 in erythropoiesis by limiting replicative stress that becomes essential in the absence of p21 and in the context of enhanced hemolysis, highlighting the potential effect that might arise from the design and use of PARP inhibitors that specifically inactivate PARP proteins.

Cell Death and Differentiation (2015) 22, 1144-1157; doi:10.1038/cdd.2014.202; published online 12 December 2014

Erythropoiesis is a complex multistage process in which multipotential hematopoietic stem cells give rise to mature red blood cells (RBCs) through coordinated programs of gene expression, proliferation and differentiation. ${ }^{1}$ The earliest committed erythroid progenitor is the erythroid burst-forming unit (BFU-E) that further differentiates through erythroid colony-forming unit (CFU-E), proerythroblasts, basophilic and polychromatophilic erythroblasts and orthochromatic erythroblasts, giving rise to reticulocytes by enucleation. Reticulocytes are then released into the circulation, where they shed their RNA and degrade mitochondria to become mature RBCs. ${ }^{2,3}$ Erythropoiesis must be tightly regulated to maintain the production of RBCs under both normal and stress conditions. This complex process is controlled intricately at each stage of the production of RBCs by multiple proteins, RNAs, DNA and chromatin modifications. ${ }^{1,4-7}$ Impairment of these regulatory pathways can lead to anemia, which has been estimated to affect nearly a quarter of the world's population. ${ }^{8}$ Moreover, defective erythropoiesis can be fatal under conditions of stress such as acute blood loss, chronic infections and myeloablative therapeutic procedures used to treat malignant diseases. Therefore, a better understanding of the molecular mechanisms controlling the production of RBCs are important to control anemia and may lead to avenues for developing new therapeutic strategies.

Poly(ADP-ribose) polymerase-2 (PARP-2) belongs to a family of enzymes that catalytically cleave $\beta$-nicotinamide adenine dinucleotide $\left(\beta-N A D^{+}\right)$and transfer the ADP-ribose moiety to acceptor residues of target proteins, modifying their functional properties by creating long chains of poly(ADPribose). ${ }^{9,10}$ This modification is a dynamic process, as indicated by the short half-life of the ADP-ribose polymer, which is rapidly subjected to degradation by the poly(ADPribose)-glycohydrolase and ADP-ribosyl-hydrolases. ${ }^{9}$ Among the PARP proteins, PARP-1, PARP-2 and PARP-3 enzymatic activities are stimulated by DNA strand interruptions targeting mainly proteins involved in chromatin structure, DNA metabolism and repair, suggesting a role for these proteins in the cellular response to DNA damage and maintenance of

${ }^{1}$ Cancer Research Program, Hospital del Mar Medical Research Institute (IMIM), Barcelona, Spain; ${ }^{2}$ Barcelona Biomedical Research Park (PRBB), Barcelona, Spain; ${ }^{3}$ CIBERehd, Barcelona, Spain; ${ }^{4}$ Genomic Instability Group, Spanish National Cancer Research Centre (CNIO), Madrid, Spain; ${ }^{5}$ Deparment of Pathology, Hospital del Mar, Barcelona, Spain; ${ }^{6}$ Division of Developmental Immunology, Biocenter, Innsbruck Medical University, Innsbruck, Austria; ${ }^{7}$ Biotechnology and Cell Signaling, UMR7242CNRS, Laboratory of Excellence Medalis, ESBS, Illkirch, France and ${ }^{8}$ Department of Immunology, Hospital del Mar, Barcelona, Spain

${ }^{*}$ Corresponding author: J Yélamos, Department of Immunology, Cancer Research Program, IMIM, Barcelona Biomedical Research Park (PRBB), Hospital del Mar, C/ Dr. Aiguader, 88, Barcelona 08003, Spain. Tel: +34 93 3160411; Fax: +34 93 3160410; E-mail: jyelamos@imim.es

${ }^{9}$ These authors contributed equally to this work.

Abbreviations: PARP, poly(ADP-ribose)polymerase; EPO, erythropoietin; RPA, replication protein A; RBCs, red blood cells; BFU-E, erythroid burst-forming unit; CFU-E, erythroid colony-forming unit; $\beta-\mathrm{NAD}^{+}, \beta$-nicotinamide adenine dinucleotide; AID, activation-induced cytidine deaminase; RS, replicative stress; MCH, mean corpuscular hemoglobin; MCHC, mean concentration of corpuscular hemoglobin; MCV, mean corpuscular volume; BM, bone marrow; BrdU, 5-bromodeoxyuridine; ATR, ataxiatelangiectasia mutated and Rad3 related; ATM, ataxia-telangiectasia mutated; GSEA, gene set enrichment analysis; PHZ, phenylhydrazine

Received 27.6.14; revised 04.10.14; accepted 05.11.14; Edited by RA Knight; published online 12.12 .14 

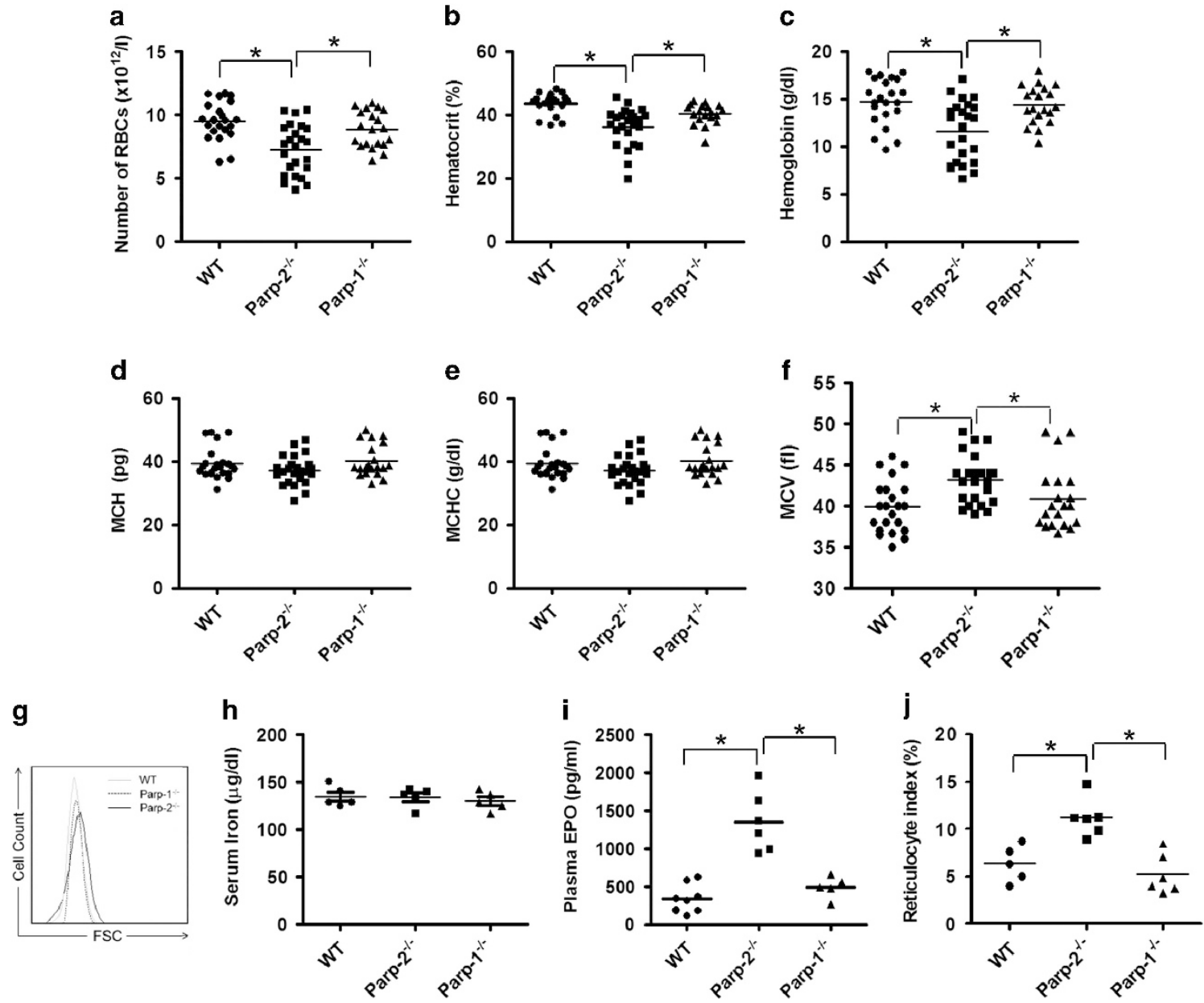

Figure 1 Analysis of erythrocyte parameters of peripheral blood revealed anemia in Parp- $2^{-1-}$, but not in Parp- $1^{-1-}$, mice. (a) Number of RBCs, (b) hematocrit, (c) hemoglobin levels, (d) MCH, (e) MCH concentration (MCHC) and (f) MCV in peripheral blood from wild-type (WT), Parp- $2^{-1-}$ and Parp- $1^{-1-}$ mice were determined on an Abacus Junior Vet Hematology Blood Analyzer. (g) Cell size of RBCs derived from WT, Parp- $1^{-1-}$ and Parp- $2^{-1-}$ mice was determined by FSC measurement by FACS. Representative histograms are shown. At least five mice per genotype were analyzed. (h) Serum iron levels, (i) plasma EPO levels and (j) reticulocyte index were determined in WT, Parp- $2^{-1-}$ and Parp $-1^{-1-}$ mice. Each symbol represents a single mice, with the bar indicating the mean. *Statistically significant difference $(P<0.05)$

genomic stability. ${ }^{9,11,12}$ Accordingly, PARP inhibitors that compete with $\beta-\mathrm{NAD}^{+}$at the highly conserved enzyme's active site arise as new potential therapeutic drugs used for chemoand radiopotentiation and for the treatment of cancers with specific DNA repair defects where they can trigger synthetic lethality as single agents. ${ }^{13}$ Despite increasing biochemical and structural evidences suggesting specific roles for these proteins in the DNA-damage response, PARP inhibitors currently in clinical trials are still unable to discriminate individual PARP-isoforms. ${ }^{14}$ For instance, PARP-2 can become selectively activated by specific stimuli, ${ }^{15}$ has particular targets and/or interacts with specific protein partners, ${ }^{16-18}$ suggesting functions independent of PARP-1 that have started to emerge. Notably, PARP-2, but not PARP-1, has been shown to be essential for T-cell development and genomic stability in thymocytes, ${ }^{19,20}$ spermatogenesis, ${ }^{21}$ suppression of activation-induced cytidine deaminase (AID)induced $\mathrm{IgH} / \mathrm{c}$-myc translocation, ${ }^{22}$ adipocyte differentiation, ${ }^{23}$ SIRT1 expression, ${ }^{24}$ cholesterol homeostasis ${ }^{25}$ and in the DNA-damage response of hematopoietic stem/progenitor cells, preserving hematopoiesis under conditions of irradiation stress. ${ }^{26}$ Understanding the role of individual PARP proteins in physiology and under pathological conditions will provide invaluable clues to design new therapeutic approaches targeting this family of enzymes.

Here, we show that deficiency of PARP-2, but not of PARP-1, in mice leads to chronic anemia at steady-state conditions. Loss of PARP-2 results in a decrease in RBC count, enlarged erythrocyte size, the presence of Howell-Jolly bodies and a reduced lifespan of circulating erythrocytes. Our results reveal that this deficiency is associated with an accumulation of replicative stress (RS) in erythroid progenitors, which limits their expansion through the activation of cellcycle checkpoints and apoptotic mediators. Finally, we show that this protective function of PARP-2 becomes essential in the absence of p21 and in the context of enhanced hemolysis. Our findings establish a pivotal, nonredundant role of PARP-2 in erythropoiesis that might impact on the development of PARP-centered therapies. 


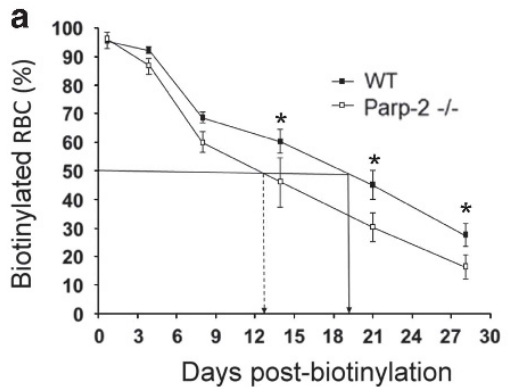

C

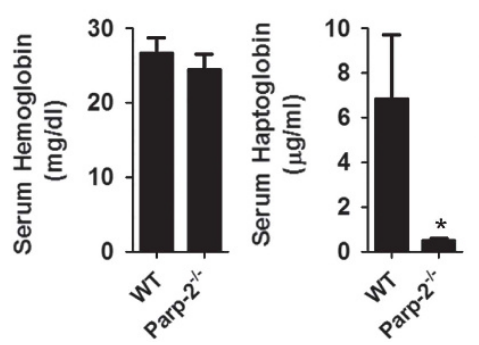

b

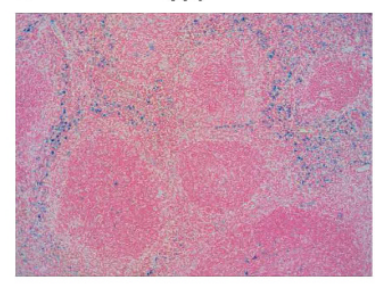

d
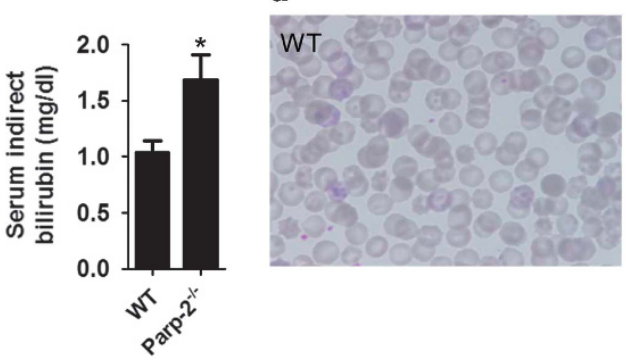

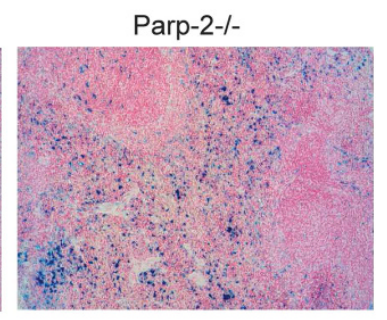

Parp-2-I-

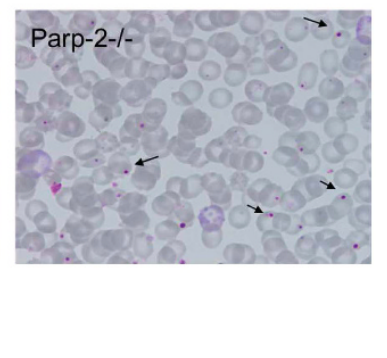

Figure 2 PARP-2-deficient erythrocytes show reduced lifespan, increased extravascular hemolysis and the presence of Howell-Jolly bodies. (a) Parp-2 ${ }^{-1-}$ erythrocytes show a reduced half-life compared with wild-type (WT) mice. RBCs from WT and Parp-2 ${ }^{-1-}$ mice were biotin labeled in vivo. Sixteen hours after injection, $95 \%$ of RBCs from both genotypes were labeled. Animals were bled at different time points, and the RBCs were stained for streptavidin and Ter119, and analyzed by flow cytometry. Values represent the mean \pm S.E.M. of the percentage of biotinylated RBCs at different time points obtained in two independent experiments, including at least five mice per genotype in each individual experiment. (b) Representative microscopy picture showing iron deposits in Parp- $2^{-1-}$ spleen, visualized by Perls's staining. (c) Serum hemoglobin, haptoglobin and indirect bilirubin levels in WT and Parp-2 $2^{-1}$ mice. Values represent the mean \pm S.E.M. obtained from at least six mice per genotype. (d) Representative microscopy picture showing micronuclei (Howell-Jolly bodies) on RBC smears from Parp- $2^{-1-}$ mice, visualized by Giemsa staining. *Statistically significant difference $(P<0.05)$

\section{Results}

\section{Loss of PARP-2, but not loss of PARP-1, causes anemia} in mice. Hematological analysis of PARP-2-deficient $\left(\right.$ Parp- $\left.2^{-1-}\right)$ mice at steady-state conditions revealed anemia as shown by a significant decrease in the number of RBCs, hematocrit and hemoglobin levels compared with wild-type littermate controls (Figures 1a-c). The mean corpuscular hemoglobin $(\mathrm{MCH})$ and the mean concentration of corpuscular hemoglobin $(\mathrm{MCHC})$ were similar in wild-type and Parp$2^{-1-}$ mice (Figures $1 \mathrm{~d}$ and e), indicating that the reduction in hemoglobin levels detected in Parp-2 $2^{-/}$mice was caused by a decrease in RBC counts. We also observed a considerable increase in the mean corpuscular volume (MCV) of RBCs from Parp-2 ${ }^{-1-}$ compared with wild-type mice (Figure 1f). Consistently, flow cytometry data showed significant increased forward light scatter in Parp- $2^{-/-}$RBCs, providing additional evidence for increased RBC size in Parp- $2^{-/-}$mice (Figure 1g). Noteworthy, the anemia phenotype was not observed in Parp-1 $1^{-1-}$ mice (Figure 1). Moreover, this phenotype was restricted to the erythroid compartment as white blood cell and platelet counts were normal in Parp- $2^{-/-}$ and Parp $-1^{-/}$mice (Supplementary Figure S1). Altogether, these data indicate that PARP-2, but not PARP-1, deficiency in mice leads to anemia despite normal serum iron levels (Figure 1h).

Anemia is known to trigger a compensatory response through the increase of erythropoietin (EPO) levels in plasma. The raised EPO promotes recovery from anemia by acting on erythroid progenitors in bone marrow (BM) and spleen, stimulating them to increase the rate at which RBCs are generated. ${ }^{27}$ Accordingly, elevated EPO levels are often associated with an increased number of circulating reticulocytes, which allows an assessment of erythropoietic rate. In agreement with this, we found an increase in EPO plasma levels in Parp-2 $2^{-1-}$ mice (Figure 1i), which associated with a 2.6-fold increase in their reticulocyte index (Figure 1j), reflecting the degree of compensatory response of Parp- $2^{-/-}$ hematopoietic tissues to anemia. However, the compensatory EPO response and the ensuing increase in circulating reticulocytes, which might account for the increased RBC size, are insufficient to avoid anemia in Parp- $2^{-/-}$mice.

Shortened RBC lifespan in PARP-2-deficient mice. The anemia observed in Parp- $2^{-1-}$ mice could be due to increased RBC destruction, faulty RBC production or a combination of both processes. To determine the RBC lifespan in vivo, we monitored the disappearance of biotinylated RBCs at different time points. RBCs circulate for about 40 days in mice until they are taken up by splenic macrophages. ${ }^{28}$ Consistently, the time required for $50 \%$ of labeled erythrocytes to be lost from wild-type mice was 19 days. However, the half-life of labeled erythrocytes decreased to 13 days in Parp-2 $2^{-/-}$mice (Figure 2a and Supplementary Figure S2). Hence, the absence of PARP-2 causes significant premature loss of circulating RBCs. In agreement, Perls-stained spleen sections from Parp- $2^{-/-}$ mice showed a marked increase in iron deposits compared with the wild-type mice (Figure 2b). Increased extravascular hemolysis of RBCs in PARP-2-deficient mice is also revealed by decreased serum haptoglobin, increased serum indirect bilirubin and normal serum hemoglobin (Figure 2c). 
The excessive removal of Parp- $2^{-/-}$RBCs might imply that they are damaged. ${ }^{29,30}$ Indeed, analysis of peripheral blood smears revealed a striking accumulation of micronuclei (Howell-Jolly bodies) on PARP-2-deficient RBCs (12.5\%) (Figure 2d). The presence of Howell-Jolly bodies in PARP-2deficient RBCs is consistent with the segregation defects that arise in response to RS. ${ }^{31-33}$ Thus, defective erythropoiesis, which might be due to the accumulation of RS on erythroid progenitors, could also be involved in the anemia observed in Parp- $2^{-1-}$ mice.

PARP-2-deficient mice show impaired differentiation of erythroid cells. To evaluate the impact of PARP-2deficiency on erythroid progenitor development, we tested the ability of wild-type and Parp- $2^{-1-}$ cells from BM and spleen to form BFU-E and CFU-E in methyl cellulose. In these assays, Parp- $2^{-1-}$ cells from both BM and spleen displayed a significant decreased capacity to form BFU-E and CFU-E than wild-type cells, suggesting an early defect of PARP-2-deficient erythroid progenitors (Figure 3a).
To further characterize the role of PARP-2 in erythropoiesis at later stages, we also examined erythroid maturation in BM and spleen using the expression of the cell-surface markers Ter119 and CD71 and the forward scatter (FSC) parameter ${ }^{34}$ (Supplementary Figure S3A). We observed an increase in both the percentage and the absolute number of Ter $119^{+}$cells in BM and spleen of Parp- $2^{-/-}$compared with wild-type mice (Figures $3 \mathrm{~b}$ and $\mathrm{c}$ ). The Ter $119^{+}$compartment showed a significant increase in the number of proerythroblasts (proE: Ter119 ${ }^{\text {med }} \mathrm{CD} 71^{\text {high }}$ ), basophilic erythroblasts (Ery.A: Ter119 ${ }^{\text {high }}$ $\mathrm{CD} 71^{\text {high }} \mathrm{FSC}^{\text {high }}$ ) and orthochromatic erythroblasts (Ery.C: Ter1 $19^{\text {high }} \mathrm{CD} 71^{\text {low }} \mathrm{FSC}{ }^{\text {low }}$ ) in both BM and spleen from Parp$2^{-1-}$ compared with wild-type mice (Figure 3d). An increase in the number of late basophilic and polychromatophilic (Ery.B: Ter119 ${ }^{\text {high }}$ CD71 ${ }^{\text {high }} \mathrm{FSC}{ }^{\text {low }}$ ) was also observed, albeit statistically significant differences were not achieved in BM (Figure 3d). The imbalanced ratio of Ery.A/Ery.B, as an index of maturation, in BM and spleen from Parp- $2^{-/-}$mice points toward a partial block in the transition from immature to mature RBC precursors (Figure $3 e$ ), suggesting that erythropoiesis in

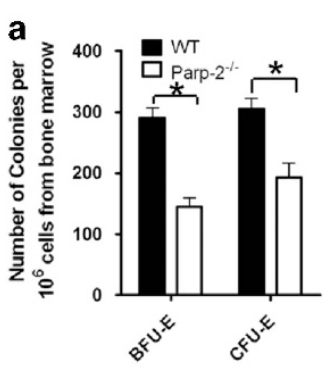

b

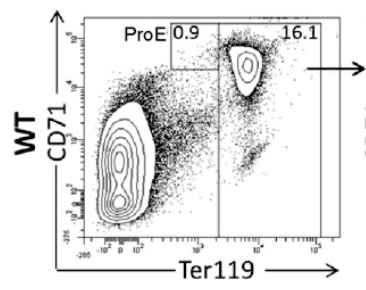

Bone Marrow

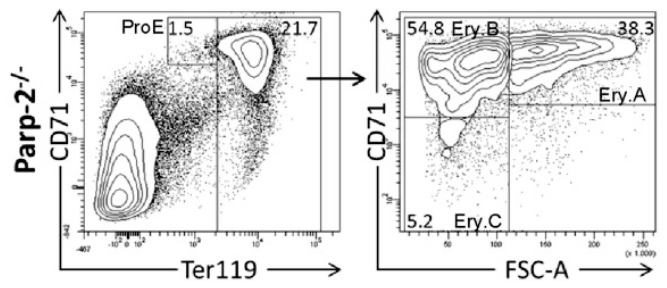

Spleen
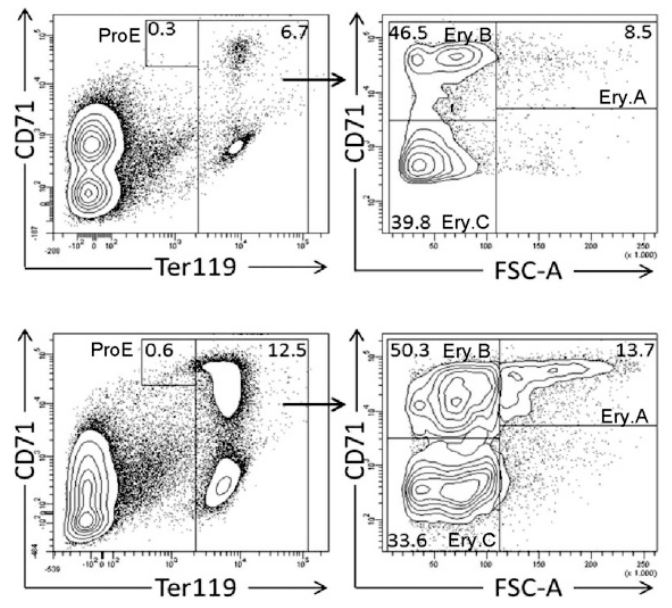
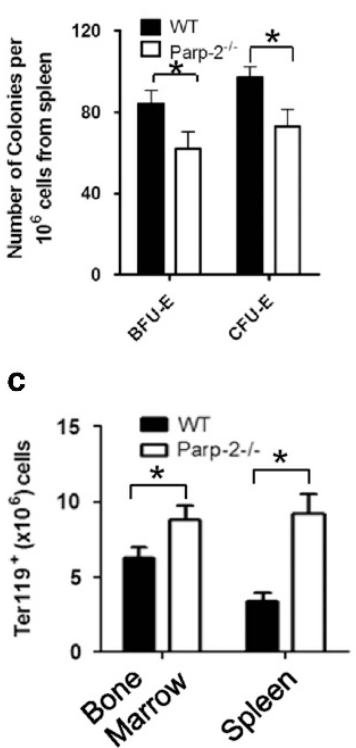

d

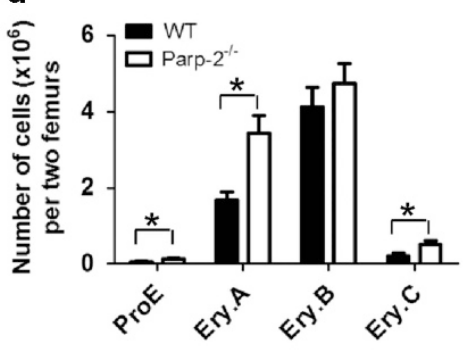

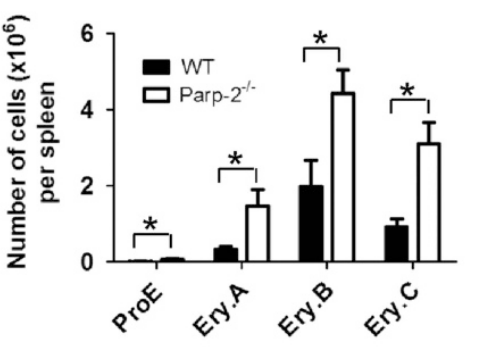

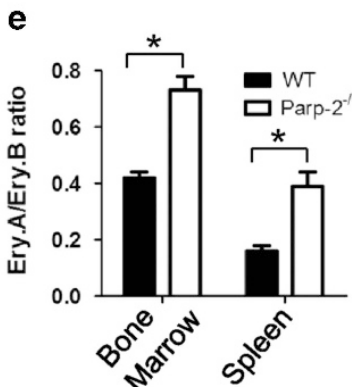

Figure 3 Impaired erythropoiesis and cell-cycle alterations in Parp-2 $2^{-1-}$ mice. (a) Colony-forming units in BM and spleen cells from wild-type (WT) and Parp-2 $2^{-1-}$ mice. Values represent the mean \pm S.E.M. from three independent experiments including at least three mice of each genotype per experiment. (b) Representative dot-plots showing erythroblast differentiation in BM and spleen from WT and Parp- $2^{-/}$mice, defined by staining for CD71 and Ter119 differentiation markers and FSC distribution. Percentage of cells in the individual sub-populations is indicated in each quadrant. Enucleated cells were lysed using ACK lysis buffer. (c) Graph showing the absolute number of Ter119 ${ }^{+}$and (d) ProE, Ery.A, Ery.B and Ery.C cells in BM and spleen from WT and Parp-2 ${ }^{-1-}$ mice. (e) Ery.A/Ery.B ratio in BM and spleen from WT and Parp-2 ${ }^{-1-}$ mice. (f) Representative cell-cycle profiles of WTand Parp- $2^{-1-}$ erythroid progenitor cells. BM cells were isolated from mice that were injected $1 \mathrm{~h}$ earlier with BrdU, surface stained for CD71 and Ter119, to define erythroblast subsets as indicate above, and analyzed for BrdU incorporation and DNA content (4',6-diamidino-2-phenylindole (DAPI) staining) in each population. The percentage of cells in each quadrant represents the mean from at least six mice in each group. (g) Graph showing the percentage of cells that are in G0/G1, S and G2/M phases of cell cycle. Values represent the mean \pm S.E.M. obtained from at least six mice per genotype. *'Statistically significant difference $(P<0.05)$ 
f
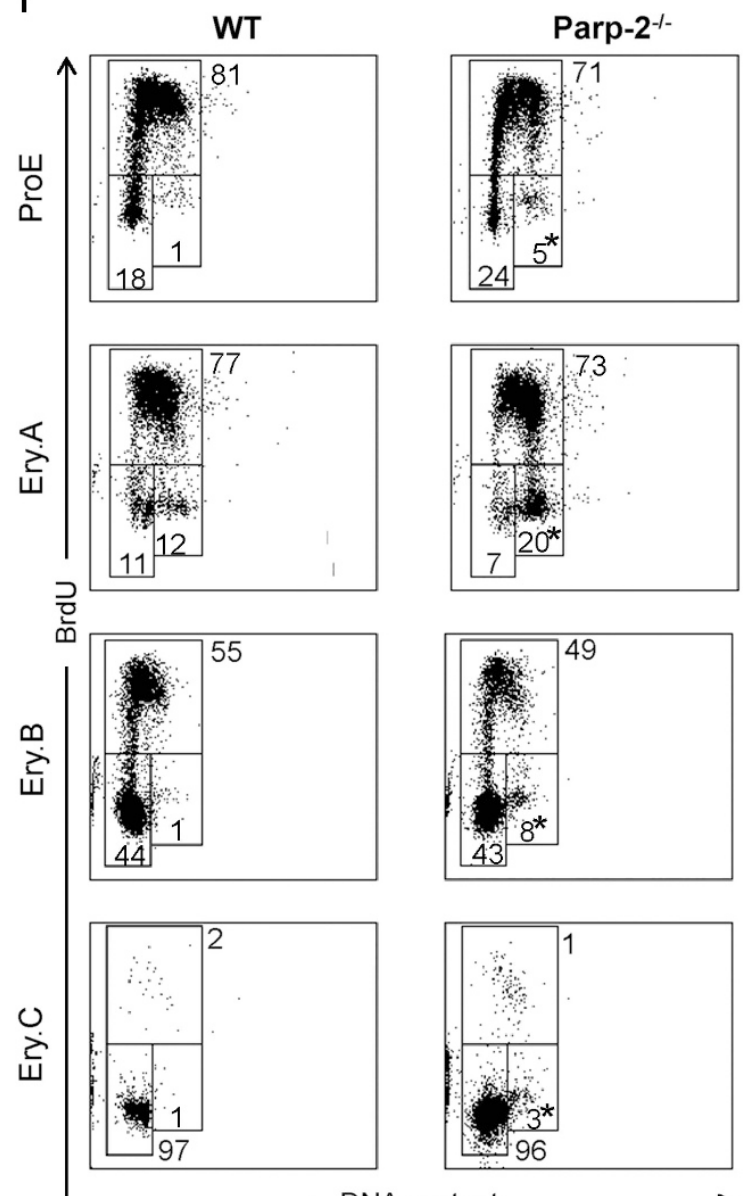

g

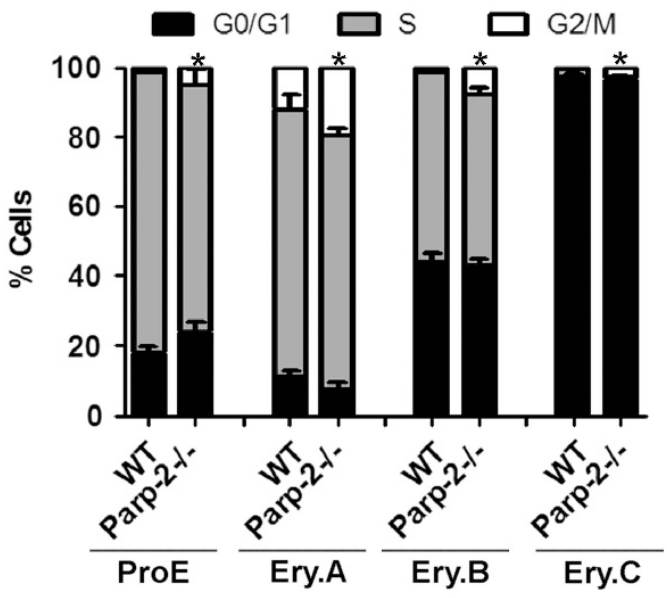

Figure 3 (Continued)

Parp- $2^{-/-}$mice may not provide adequate reticulocytosis to compensate the massive hemolysis.

To determine possible causes behind the limited functionality of PARP-2-deficient erythroid progenitors, we analyzed their cell-cycle distribution. BM cells were isolated $1 \mathrm{~h}$ after 5-bromodeoxyuridine (BrdU) injection of mice, stained for surface expression of CD71 and Ter119 and the cell-cycle status were assessed by BrdU staining to detect DNA synthesis and with DAPI to gauge the amount of DNA per cell (Supplementary Figure S3B). Flow cytometry data revealed that Parp- $2^{-/-}$erythroid progenitors exhibit a G2/M cell-cycle arrest (Figures $3 f$ and $\mathrm{g}$ ). In contrast, no alteration of either erythroid differentiation or the cell-cycle profile was observed in PARP-1-deficient erythroid progenitors (Supplementary Figure S4). Similar to the previously mentioned accumulation of micronuclei, the accumulation of cells in $\mathrm{G} 2 / \mathrm{M}$ is reminiscent of $\mathrm{RS}$.

RS limits the functionality of PARP-2-deficient erythroid progenitors. To directly evaluate the presence of RS in Parp $-2^{-/-}$erythroblasts, we first used flow cytometry to monitor the phosphorylation of histone H2AX $(\gamma-\mathrm{H} 2 \mathrm{AX})$, a sensitive indicator of DNA injury. ${ }^{35}$ The percentage of $\gamma$-H2AX-positive cells was significantly higher in Parp- $2^{-1-}$ erythroblasts than that noted in wild-type cells (Figures $4 \mathrm{a}$ and $b$ ). Noteworthy, the increase on $\gamma-\mathrm{H} 2 \mathrm{AX}$ was limited to $\mathrm{S} /$ $\mathrm{G} 2$ cells, a hallmark of $\mathrm{RS}^{36,37}$ (Figure 4c). Next, we monitored on sorted Ter $119^{+}$erythroblasts the phosphorylation status of $\mathrm{CHK} 1$ on S345, a target of the ataxiatelangiectasia mutated (ATM) and Rad3 related (ATR) kinase that coordinates the response to RS. ${ }^{38}$ Our results revealed increased levels of phosphorylation of CHK1 in Parp- $2^{-/-}$ compared with wild-type cells (Figure 5). Of note, ATR has also been demonstrated to phosphorylate H2AX during replication fork blockage. ${ }^{39}$ We have also observed an increased level of replication protein A (RPA) phosphorylation on S4/S8 (Figure 5), triggered by ATM and DNA-PK (DNAdependent protein kinase) kinases in response to RS. ${ }^{40}$

Gene expression analyses of PARP-2-deficient erythroblasts reveal the activation of p53-dependent DNA-damage response pathways. To gain further insights into the effect of PARP-2 deficiency on erythroblasts, we performed microarray on sorted $\mathrm{Ter}^{119^{+}} \mathrm{BM}$ erythroblasts from Parp- $2^{-/-}$and wild-type mice. Clustering of all genes that changed between Parp- $2^{-/-}$and wild-type erythroblasts is represented in Supplementary Figure S5. Gene set enrichment analysis (GSEA) ${ }^{41}$ showed a significant 
a

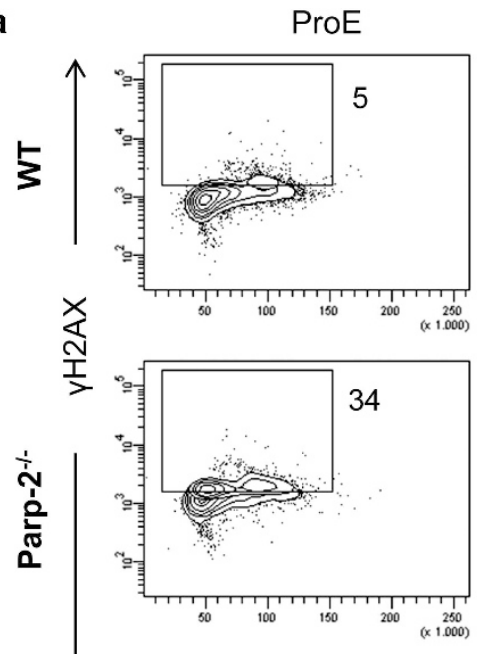

Ery.A
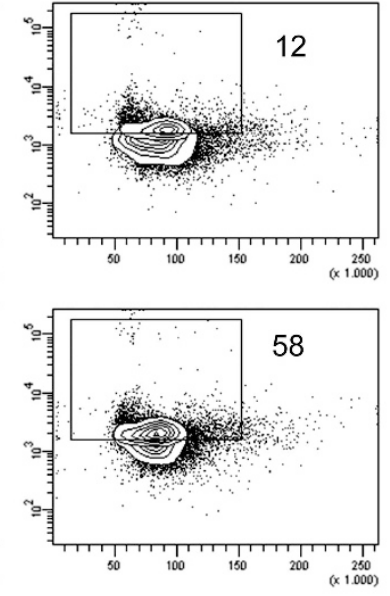

Ery.B
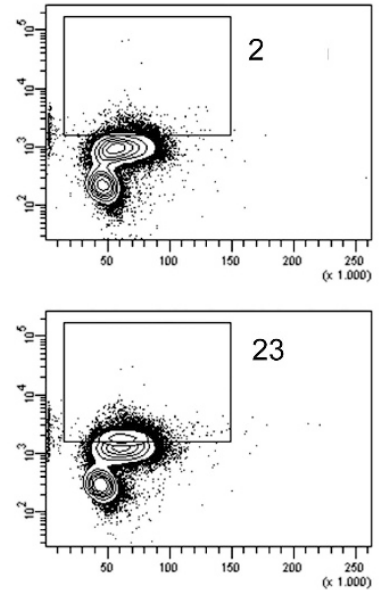

Ery.C
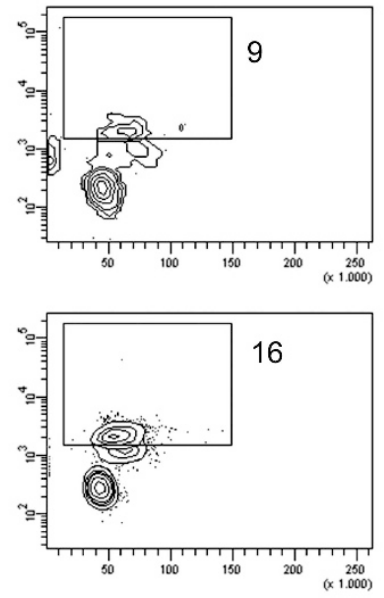

DNA content

b

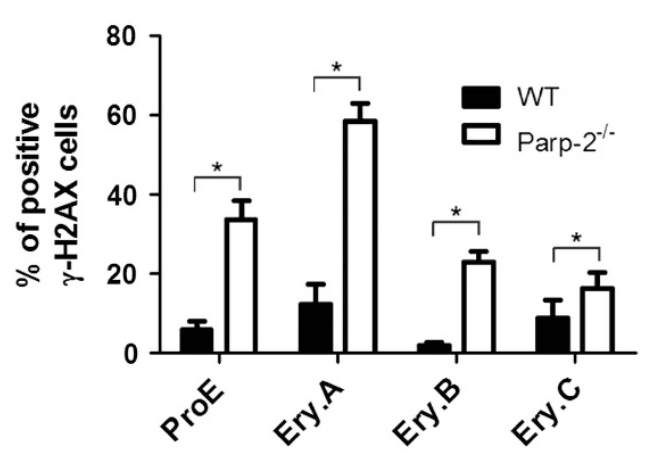

c

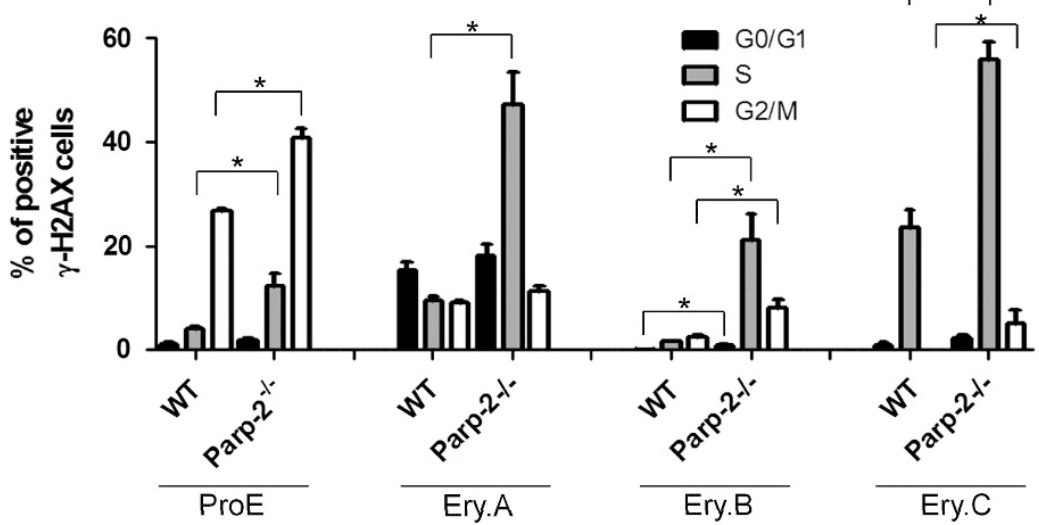

Figure 4 Accumulation of DNA damage in Parp- $2^{-/-}$erythroblasts. (a) Representative staining profiles of $\gamma$-H2AX in ProE, Ery.A, Ery.B and Ery.C population derived from wild-type (WT) and Parp-2 $2^{-1-}$ mice, defined by Ter119 and CD71 staining and FSC distribution. (b) Graph showing the percentage of $\gamma$-H2AX-positive cells in each stage of erythroid development. Bars represent the mean \pm S.E.M. obtained from at least six mice per genotype. (c) Graph showing the percentage of $\gamma$-H2AX-positive cells in G0/G1, S and G2/M phases of cell cycle in erythroblasts from WT and Parp- $2^{-1-}$ mice, at different stages of differentiation. BM cells were isolated from mice that were injected $1 \mathrm{~h}$ earlier with BrdU, surface stained for CD71 and Ter119, to define each stage of erythroid development, and analyzed for BrdU incorporation, $\gamma$-H2AX presence and DNA content (4',6-diamidino-2-phenylindole (DAPI) staining). Bars represent the mean \pm S.E.M. obtained from at least six mice per genotype. *Statistically significant difference $(P<0.05)$

enrichment for p53-dependent signaling pathways in Parp- $2^{-/-}$compared with wild-type erythroblasts (Figures $6 a$ and b). For instances, p53-dependent cell-cycle regulator genes, such as p21 (Cdkn1a), Ccng1, Tp53inp1, as well as the cell-cycle associated gene, Ccnb1ip1, were markedly increased in Parp- $2^{-/-}$erythroblasts (Figures $6 \mathrm{~b}$ and $\mathrm{c}$ ).

In addition, proapoptotic p53-dependent genes (Bax, Puma and Phlda3) were also overexpressed in Parp- $2^{-1-}$ cells (Figures $6 b$ and $c$ ). Accordingly, higher levels of apoptosis, measured by immunostaining of active caspase-3 and immunofluorescence microscopy, were also observed in Parp- $2^{-1-}$ compared with wild-type erythroblasts (Figure $6 \mathrm{~d}$ ). These data prompted us to perform a genetic rescue experiment exploring the role of Puma in DNA-damagemediated apoptosis of erythroblasts, caused by PARP-2 deficiency. This analysis suggested that loss of immature erythroblasts was due to p53-mediated apoptosis as, remarkably, Puma deficiency restores hematocrit in Parp $-2^{-/-}$mice to wild-type levels (Figure 6e).
Impaired fetal liver erythropoiesis in Parp- $2^{-1-}$ embryos is amplified by loss of p21, resulting in lethality of newborn double-deficient mice. To investigate the functional interaction between PARP-2 and the cell-cycle regulator p21, we crossed Parp- $2^{-/-}$with $\mathrm{p} 21^{-/-}$mice and the resulting Parp $-2^{+/-} \mathrm{p} 21^{+/-}$mice were intercrossed in an attempt to generate PARP-2-deficient mice lacking p21. Interestingly, PARP-2/p21-double-null mice died at newborn stage (Figure $7 \mathrm{a}$ and Supplementary Table S1). At 14.5 days postcoitum (E14.5), PARP-2/p21-double-deficient embryos were pale (Figure 7b), suggesting exacerbated anemia. Noteworthy, PARP-1/p21-double-null adult mice were obtained at the normal Mendelian frequency (data not shown).

The fetal liver is the major tissue for definitive erythropoiesis during mid-gestation. ${ }^{1}$ Interestingly, PARP-2-null fetal liver displayed a significant decrease in cell number compared with wild-type or p21 1 - fetal liver. This phenotype was strengthened by loss of p21 (Figure 7c). Further examination of 

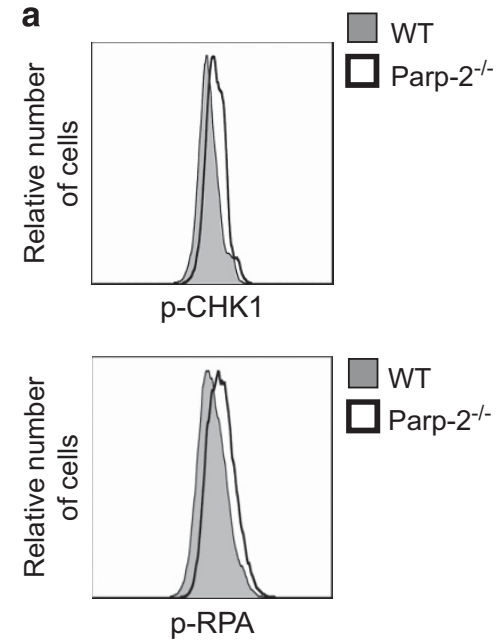

b

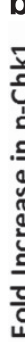

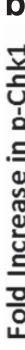
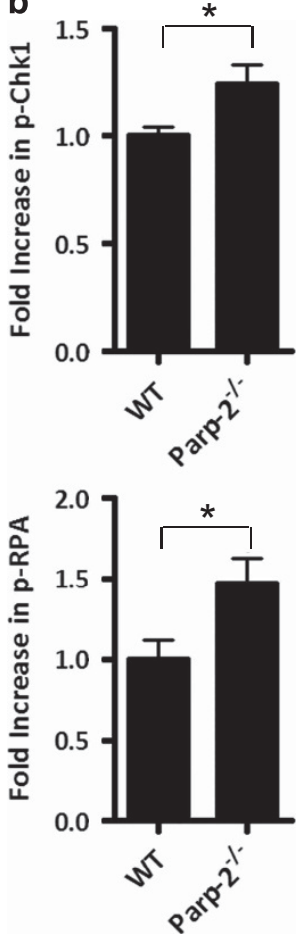

C

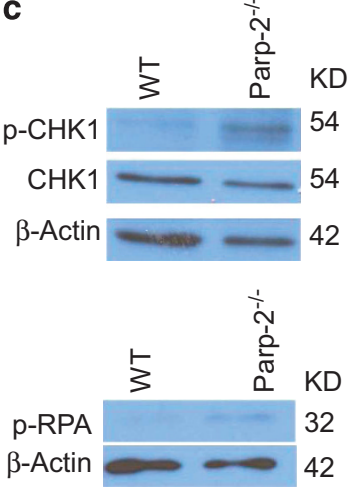

Figure 5 PARP-2 deficiency in erythroblasts induces phosphorylation of CHK1 and RPA. (a) Representative histograms showing phospho-CHK1 and phospho-RPA protein levels in erythroblasts derived from wild-type (WT) and Parp- $2^{-l-}$ mice. (b) The relative phospho-CHK1 and phospho-RPA protein levels were calculated on the basis of the mean fluorescence intensity determined by flow cytometry, and were presented as fold induction compared with WT cells. Bars represent the mean \pm S.E.M. obtained from six mice per genotype. *Statistically significant difference $(P<0.05)$. (c) Western blot expression analysis of $\mathrm{CHK} 1$, phospho-CHK1, phospho-RPA and $\beta$-actin proteins in erythroblasts from WT and Parp- $2^{-1-}$ mice. The data are representative of two independent experiments. KD, kiloDalton

erythrocyte development by flow cytometry revealed a significant decrease in both the percentage and the absolute number of Ter $119^{+}$cells in fetal liver from Parp- $2^{-/-}$embryos that was also aggravated by the loss of p21 (Figures 7d and e). This result was confirmed by fetal liver immunostaining of Ter119 and microscopy analysis (Supplementary Figure S6). The Ter $119^{+}$compartment showed a significant decrease in the number of Ery.A and Ery. B cells in fetal liver from PARP-2/ p21-double-null embryos compared with fetal liver from wildtype, $\mathrm{p} 21^{-/-}$or Parp-2 $2^{-/-}$embryos (Figures $7 \mathrm{~d}-\mathrm{f}$ ). In addition, Parp $-2^{-/-}$fetal liver exhibited a significant decrease in the number of Ery.B cells compared with wild-type or $\mathrm{p} 21^{-1-}$ fetal liver (Figures 7d-g).

Next, we assessed the cell-cycle status of the erythroblast compartments in fetal liver from embryos of the four genotypes. We detected a G2/M arrest in the Parp- $2^{-/-}$fetal liver erythroblasts (Figures $7 \mathrm{~h}$ and $\mathrm{i}$ ), which was only slightly modified by loss of p21. However, p21 deficiency significantly altered G0/G1 cell-cycle checkpoint in Parp-2 $2^{-/-}$Ery.B and Ery.C cells (Figures $7 \mathrm{~h}$ and i). No differences in erythroblast development was observed between $\mathrm{p} 21^{-/-}$and wild-type embryos (Figure 7).

Altogether, these data suggest a functional interaction between PARP-2 and p21 in the maintenance of fetal liver erythropoiesis, characterized by a rapid growth and little reserve capacity in the erythropoietic system, ${ }^{42}$ that impact on embryonic development.
PARP-2 deficiency compromises mouse survival in the context of enhanced hemolysis. Whereas the chronic anemia present on PARP-2-deficient mice did not overtly alter their lifespan, it could have more profound defects in the context of stress-driven compensatory erythropoiesis. To test this, we challenged wild-type and Parp $-2^{-/-}$mice with phenylhydrazine $(\mathrm{PHZ})$, a compound that induces oxidative denaturation of hemoglobin resulting in a rapid destruction of RBCs and severe hemolytic anemia. ${ }^{43}$ Mice were injected with $\mathrm{PHZ}$ on days 0,1 and 3 and survival was monitored for 15 days. PARP-2 deficiency severely limited the survival in response to treatment, and eight out of nine Parp $-2^{-/}$mice died within 7 days after the first $\mathrm{PHZ}$ injection, whereas all but one control mouse survived the hemolytic challenge (Figure 8a). Accordingly, Parp- $2^{-/-}$mice exhibited a larger drop in hematocrit compared with wild-type mice on day 5 after the first $\mathrm{PHZ}$ injection (Figure 8b). Despite a higher compensatory increase in EPO levels (Figure 8c), the reticulocyte index at day 5 after the first $\mathrm{PHZ}$ injection was significantly decreased in Parp $-2^{-/-}$mice (Figure $8 d$ ), suggesting that defective erythropoiesis is responsible for the enhanced anemia-induced lethality of Parp- $2^{-/-}$mice.

To further evaluate $\mathrm{PHZ}$-induced erythropoiesis, maturation stages of erythroid cells from BM and spleen were analyzed at day 5 after the first $\mathrm{PHZ}$ injection. No differences were observed in BM and spleen cellularity between both genotypes (Figure 8e). Flow cytometric analysis revealed a 
a

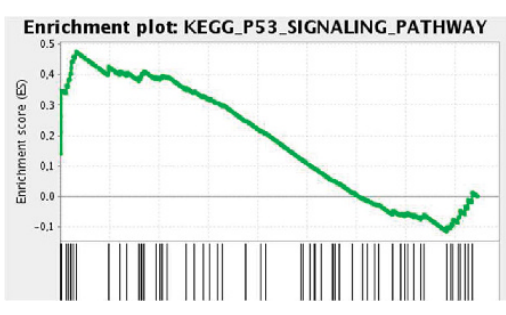

b

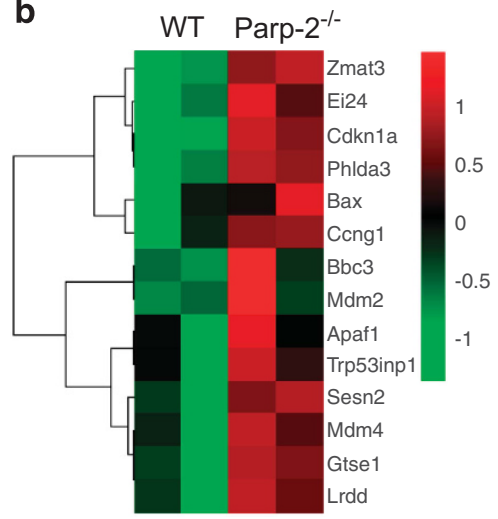

C

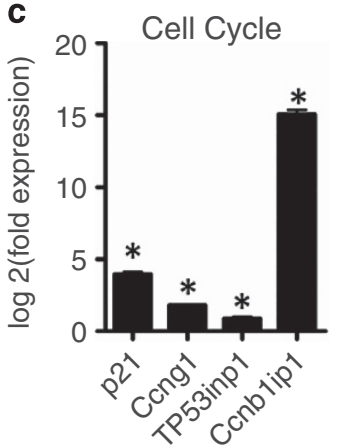

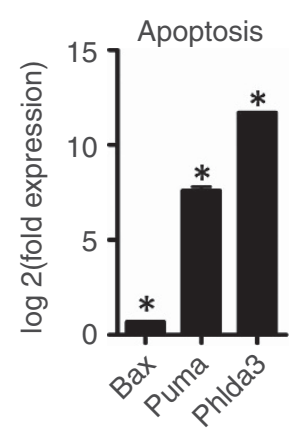

d

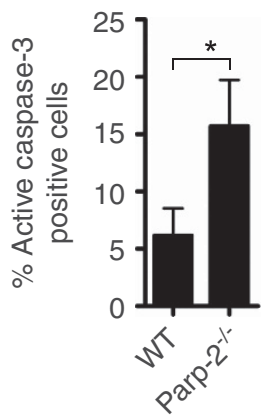

e

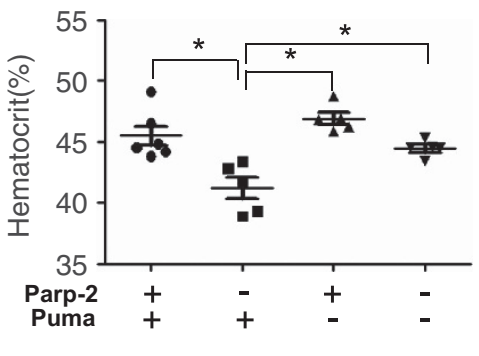

Figure 6 Activation of p53-dependent DNA-damage response in Parp-2 - / - erythroblasts. (a) Large-scale gene expression and GSEA showed a significant enrichment for p53 signaling pathways in Parp- $2^{-1-}$ compared with wild-type (WT) erythroblasts. Canonical pathways gene sets were scored using the GSEA and $P$-values were computed using 1000 permutations. Within the GSEA plots, the green line represents the sliding enrichment score and the black bars demarcate the position of the gene set members within the ranked expression data. (b) Hierarchical clustering and heatmap of gene expression in Parp- $2^{-1-}$ and WT erythroblasts using the gene set KEGG_p53_Signaling pathway. (c) Quantitative reverse transcription-PCR analysis of genes involved in cell-cycle checkpoints and apoptosis in erythroblasts. Samples were normalized according to $\beta$-actin expression levels. Results are expressed as log 2-fold expression compared with levels measured in WT cells. Values represent the mean \pm S.E.M. obtained from three independent experiments. (d) Parp- $2^{-1-}$ erythroblasts shown and increased apoptosis compared with WT cells. Bars represent the percentage of cells positive for active caspase-3, determined by fluorescent microscopy. An average of 150 cells was scored for each mouse. Values represent the mean \pm S.E.M. obtained from at least six mice per genotype. (e) Puma deficiency restores hematocrit levels in Parp- $2^{-1-}$ mice. Each symbol represents a single mice, with the bar indicating the mean. *Statistically significant difference $(P<0.05)$

significant decrease in the percentage and number of Ter119 cells in BM from Parp- $2^{-/-}$mice compared with wild-type mice, mainly due to a sharp decrease in Ery.B cell number (Figures $8 \mathrm{f}-\mathrm{h}$ ). However, a significant increase in the total number of Ter119+ cells was observed in the spleen of Parp$2^{-/-}$mice compared with wild-type mice, because of the accumulation of the more immature ProE and Ery.A erythroblasts (Figures $8 \mathrm{f}-\mathrm{h}$ ). Nevertheless, BM and spleen from Parp$2^{-/-}$-treated mice exhibit an imbalanced ratio of immature/ mature erythroblasts established by calculating the ratio of Ery.A/Ery.B numbers (Figure 8i). Altogether, our data indicate that PARP-2 becomes essential for survival in conditions that demand an increased production of RBCs.

\section{Discussion}

Although anemia is the most common blood disorder, ${ }^{8}$ the molecular mechanisms governing erythrocyte number persist largely enigmatic. Here we have shown that deletion of PARP-2, but not PARP-1, in mice leads to chronic anemia providing a novel role for PARP-2 in erythropoiesis. Anemia arises even in the presence of increased EPO levels on PARP-2-deficient mice, which reflects a futile compensatory response that fails to provide sufficient erythropoiesis. Although chronic anemia observed in PARP-2-deficient mice is compatible with life at steady-state conditions, mice died rapidly when exposed to conditions that demand a higher production of RBCs. When we examined the erythroid maturation from the proerythroblast to late orthochromatic erythroblast stage, we found that the rate of maturation of BM Parp- $2^{-/-}$erythroid cells at steady-state conditions was significantly reduced and was accompanied by an increase in the size of proerythroblasts and basophilic erythroblast populations. This might reflect a block in differentiation and subsequent apoptosis of precursor cells limiting the accumulation of damaged cells. Indeed, we observed an increased percentage of active caspase-3-positive erythroblasts in the absence of PARP-2. Moreover, the chronic anemia in Parp $-2^{-/-}$mice was abolished in a Puma-deficient background, similarly to what we previously observed in response to $\gamma$-irradiation. ${ }^{26}$

Further evidence for a role of PARP-2 in erythroid development is provided by the presence of Howell-Jolly bodies in RBCs from Parp- $2^{-/-}$mice. Howell-Jolly bodies are a marker of genotoxicity, which arise when replicating cells are subjected to chromosomal breakage or chromosomal loss by 
mitotic spindle dysfunction. ${ }^{44,45}$ Our data are thus in agreement with previous in vitro studies that proposed a role for PARP-2 in chromosome segregation during cell division. ${ }^{21,46}$
For instance, Menissier de Murcia et al. ${ }^{46}$ found a high proportion of aberrant anaphases in Parp- $2^{-1-}$ mouse embryonic fibroblasts following $\mathrm{N}$-methyl- $\mathrm{N}$-nitrosourea a

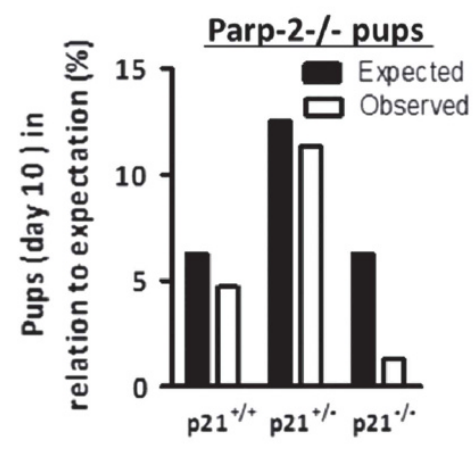

b

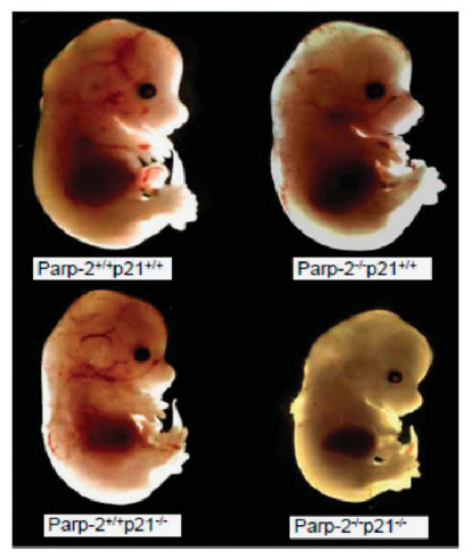

c

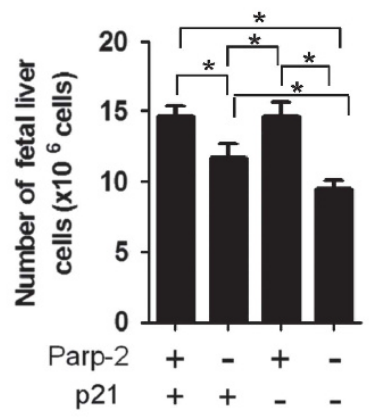

d
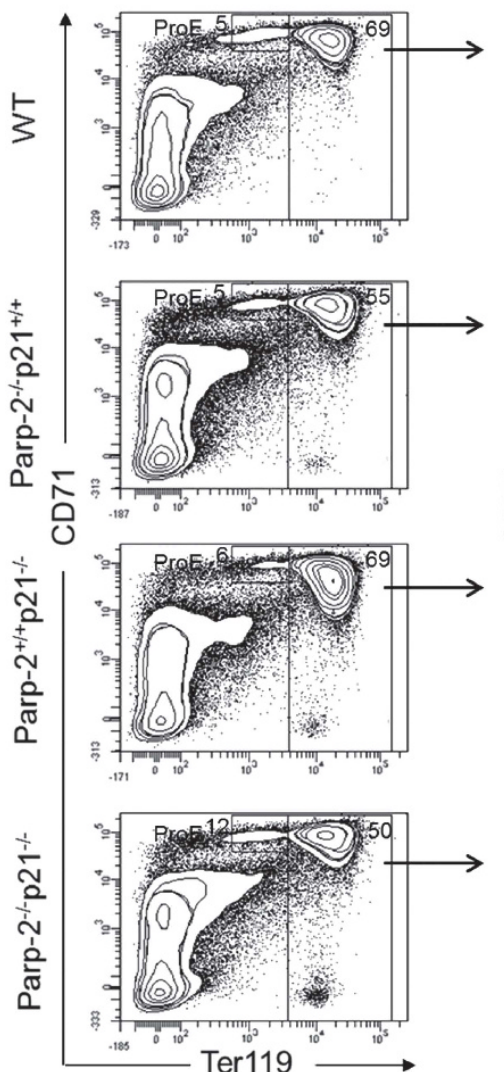

e

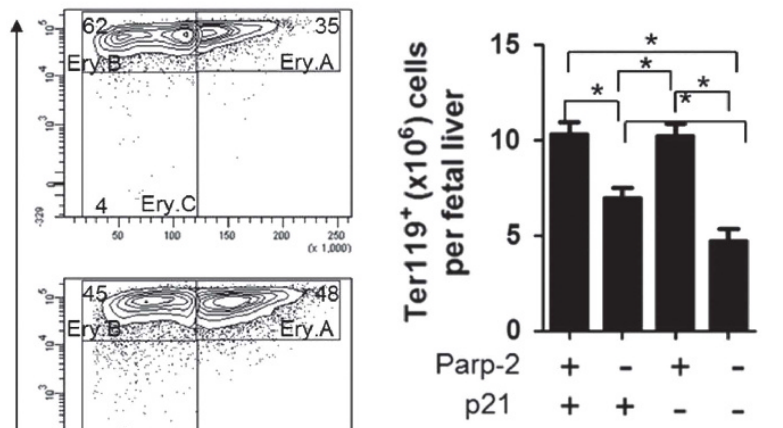

g

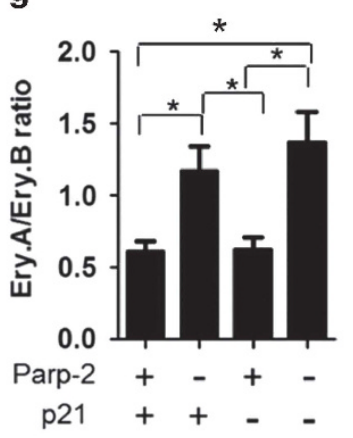

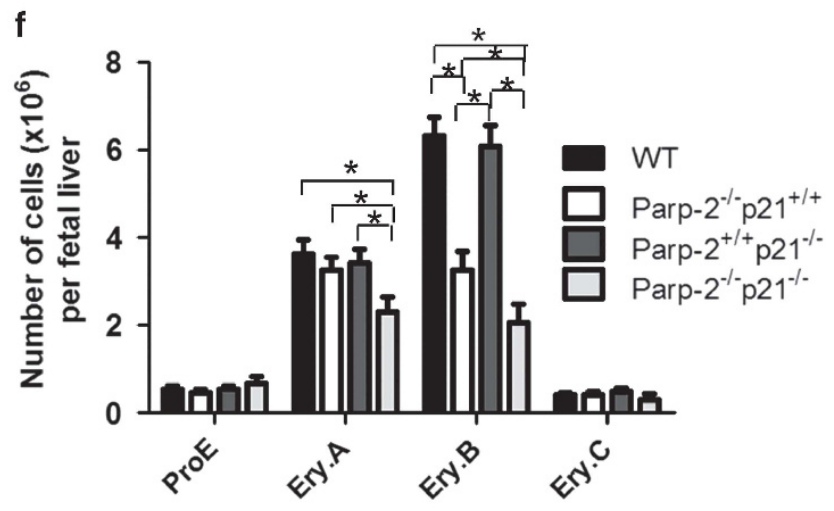

Figure 7 Functional interaction between PARP-2 and p21 in erythropoiesis. (a) The Mendelian expected frequency (black bars) and the observed frequency (white bars) of Parp-2 $2^{-1}$ pups born with different p21 status were compared in a cohort of 483 pups born from Parp- $2^{+1-}$ p2 $1^{+1-}$ intercrosses. (b) Photograph showing the morphology of Parp$2^{+/+}$p21 $1^{+/+}(\mathrm{WT})$, Parp- $-2^{-/} \mathrm{p} 21^{+/+}$, Parp- $2^{+/+} \mathrm{p} 21^{-/-}$and Parp- $2^{-/} \mathrm{p} 21^{-/-}$embryos at 14.5 days postcoitum (E14.5). (c) Total number of fetal liver cells at E14.5. Values represent the mean \pm S.E.M. obtained from at least six embryos per genotype. (d) Representative flow cytometry of nucleated fetal liver cells staining for CD71 and Ter119 differentiation markers and FSC distribution. Percentage of cells in the individual sub-populations is indicated in each quadrant. (e) Graph showing the absolute number of Ter119+, and (f) ProE, Ery.A, Ery.B and Ery.C cells in fetal liver of E14.5 embryos. (g) Ery.A/Ery.B ratio in fetal liver of E14.5 embryos. *Statistically significant difference $(P<0.05)$. (h) Representative cell-cycle profiles of WT, Parp- $2^{-1-} \mathrm{p} 21^{+/+}$, Parp- $2^{+/+} \mathrm{p} 21^{-1-}$ and Parp- $2^{-1} \mathrm{p} 21^{-1-}$ fetal liver erythroid progenitor cells. Fetal liver cells were isolated from E14.5 embryos obtained from mothers that were injected $1 \mathrm{~h}$ earlier with BrdU, surface stained for CD71 and Ter119, to define erythroblast subsets, and analyzed for BrdU incorporation and DNA content (4',6-diamidino-2-phenylindole (DAPI) staining) in each population. The percentage of cells in each quadrant represents the mean from at least three embryos in each group. *Statistically significant difference $(P<0.05)$ relative to WT. (i) Graph showing the percentage of cells that are in G0/G1, S and G2/M phases of cell cycle. Values represent the mean \pm S.E.M. obtained from at least three embryos per genotype 
h

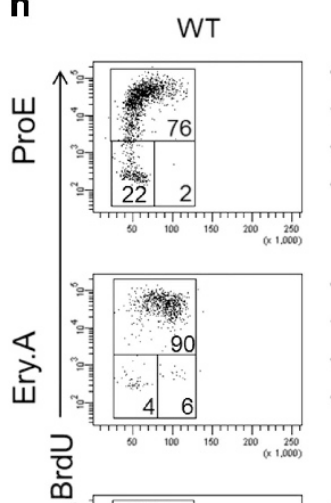

Parp $-2^{-/}$p $21^{+/+}$
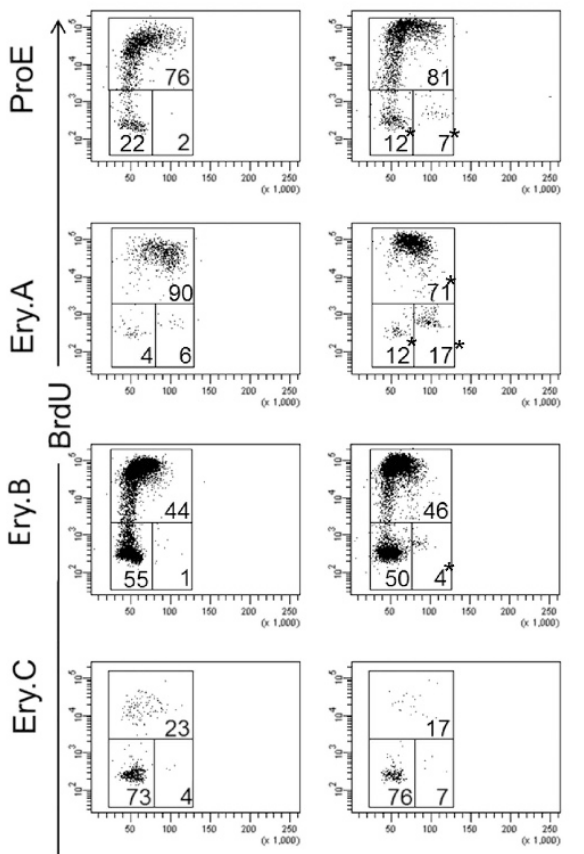

Parp- $2^{+/+}$p $21 \%$
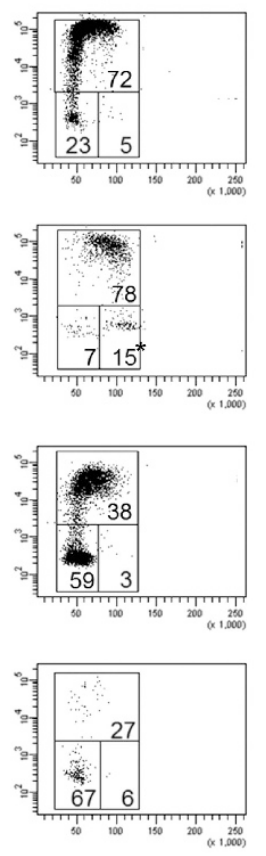

DNA content

Figure 7 (Continued)

treatment, and a significant fraction of anaphase cells contained one or more lagging (nonsegregated) chromosomes. Of note, segregation problems led to chromatid breaks, which occurred more frequently in centromeric regions in Parp $-2^{-1-}$ cells, thus providing evidence for a possible role for PARP-2 in the maintenance of centromeric heterochromatin integrity, ${ }^{47}$ and a role in accurate chromosome segregation. ${ }^{21}$ Interestingly, it was shown that RS can lead to chromosome segregation problems that can be detected as anaphase bridges and micronuclei. ${ }^{31,32}$

In agreement with a role of PARP-2 in maintaining genomic stability, ${ }^{9}$ our data indicate that PARP-2 deficiency in erythroblasts results in DNA damage, essentially occurring in replicating cells, as indicate by the accumulation of $\gamma$-H2AX in S phase. $\gamma$-H2AX formation has normally been associated with the induction of double strand breaks after exposure to DNA-damaging agents. ${ }^{48}$ However, a number of reports have shown phosphorylation of $\mathrm{H} 2 \mathrm{AX}$ after $\mathrm{RS},{ }^{49-52}$ which might be mediated in part by ATR. ${ }^{39}$ Indeed, the increased phosphorylation of CHK1 is indicative of an active RS-driven ATR response on PARP-2-deficient erythroid progenitors. This signaling cascade is associated with a transcriptional activation of p53-dependent cytostatic and apoptotic signals in Parp$2^{-1-}$ erythroblasts, although we cannot rule out that altered gene expression in Parp- $2^{-/}$cells may be caused by a direct role of PARP-2 in transcription regulation. ${ }^{53}$ Moreover, lethality of PARP-2/p21-double-null pups, due to a defect in fetal liver erythropoiesis, suggests an accumulation of RS in the absence of PARP-2. ${ }^{54,55}$ Altogether, these data suggest a critical role of PARP-2 in limiting $\mathrm{RS}$ in the highly divided erythroblast population. Remarkably, some of the described biological processes in which PARP-2, but not PARP-1, has been specifically involved are related with high proliferative cell rate such as thymopoiesis, ${ }^{19,20}$ spermatogenesis ${ }^{21}$ and hematopoiesis under stress conditions. ${ }^{26}$ This bias for pathologies on tissues with high proliferative index has previously been noted on other mutant mice with a compromised response against $\mathrm{RS}$, such as ATR-hypomorphic mice. ${ }^{56}$

Altogether, our results are consistent with a model (Supplementary Figure S7) whereby PARP-2 is specifically required to limit $R S$ in erythroid progenitors. In the absence of PARP-2, highly divided erythroblasts accumulate RS leading to genomic instability that triggers the proximal ATR/H2AX/ CHK1 DNA-damage response, which finally activates p53/p21 response resulting in G2/M arrest and apoptosis. Although damaged erythroblasts might arrive to circulation, they have a shorter lifespan. Taken together, deficient erythropoiesis and shortened RBC lifespan conduce to chronic anemia observed in Parp- $2^{-/}$mice. Whereas a compensatory increase in EPO production contributes to maintain erythropoiesis in Parp- $2^{-/-}$ mice, this response is insufficient in the context of enhanced hemolysis.

Although mounting evidence has accumulated indicating a role for PARP proteins at replication forks, most of these works were carried out with nonselective inhibitors, and pointed towards a role for PARP $-1^{57-61}$ and to less extent PARP-2 on this response. ${ }^{61}$ Moreover, all these works were carried out in vitro and to what extent this replication-linked role of PARP had in the context of a living organism remained unknown. Our results provided strong evidence that PARP-2, but not PARP-1, is required for efficient erythropoiesis in mice by limiting $R S$ on erythroid progenitors, a function that becomes essential in the context of an enhanced demand for the production of RBCs. 
a

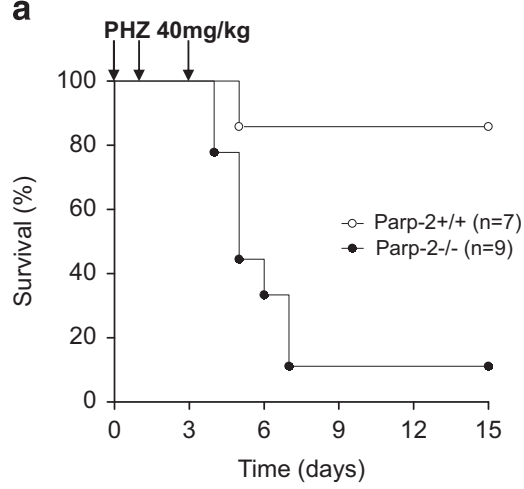

b

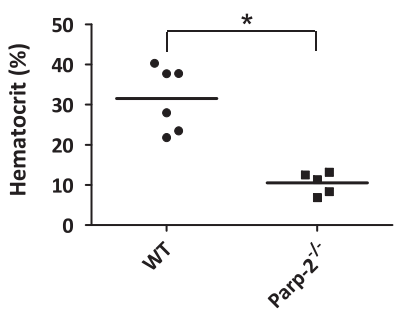

C

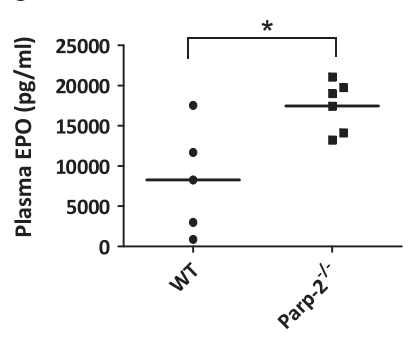

d

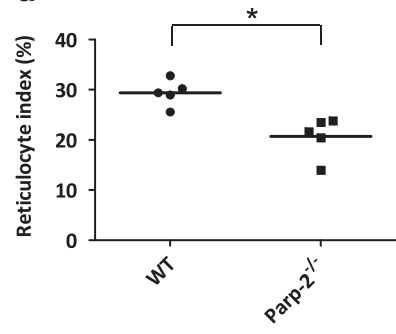

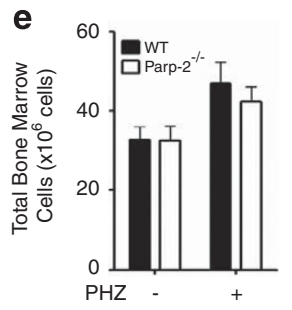

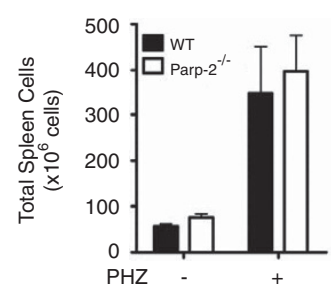

f
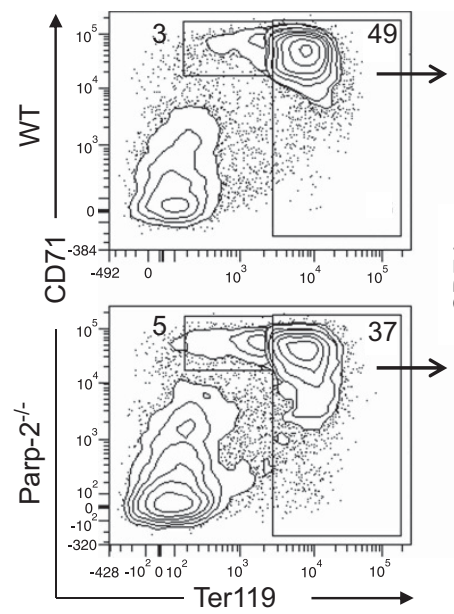

g

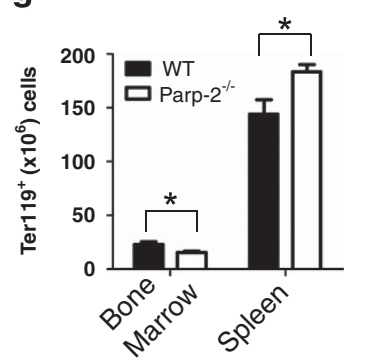

h
BoneMarrow
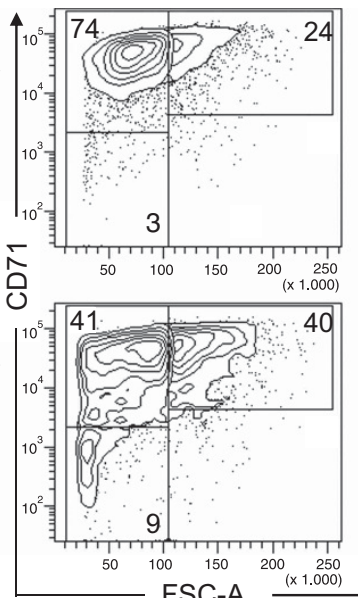

\begin{tabular}{rrr}
100 & $150 \quad 200 \quad \begin{array}{l}250 \\
(\times 1.000)\end{array}$ \\
\hline
\end{tabular}
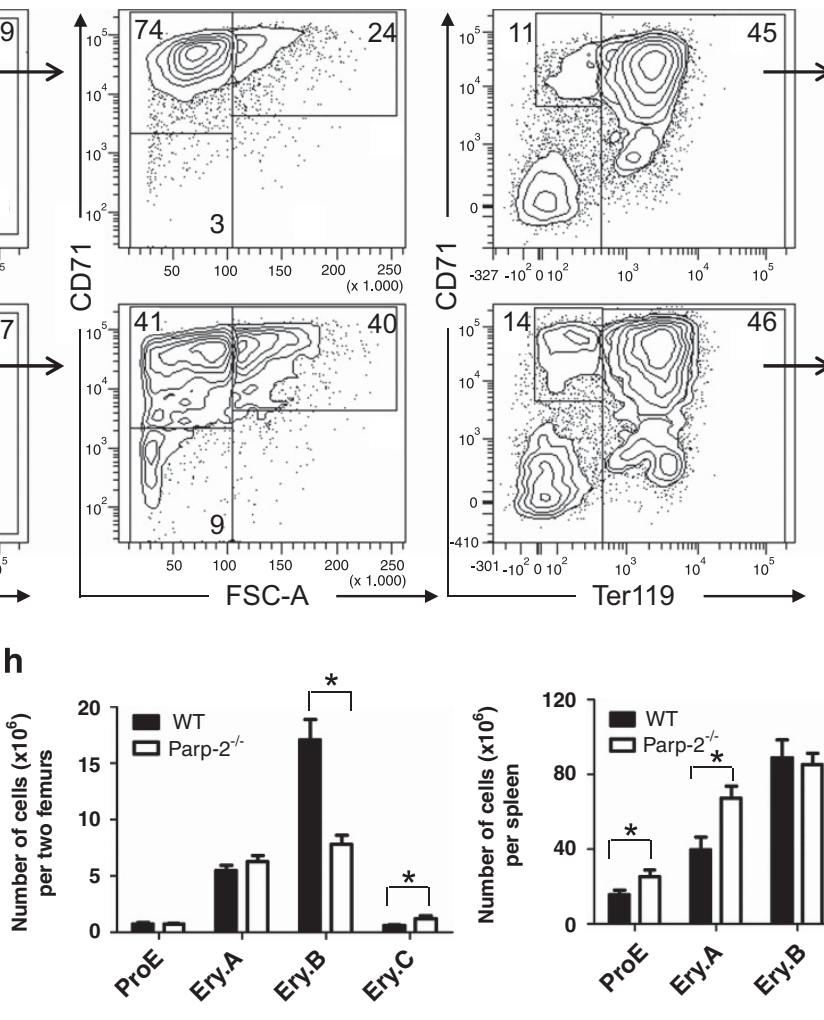

Spleen
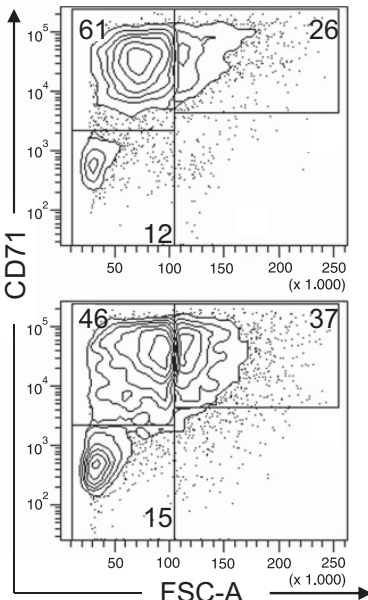

Figure 8 PARP-2-deficient mice are very sensitive to stress-induced erythropoiesis. (a) Kaplan-Meier survival curves of Parp-2 $2^{-/-}$and wild-type $\left(\right.$Parp-2 ${ }^{+/+}$) mice after receiving three intraperitoneal injections of $\mathrm{PHZ}$ (40 mg/kg mice) at day 0,1 and 3. (b) Hematocrit, (c) plasma EPO levels and (d) reticulocyte index were determined at day 5 postadministration of PHZ. Each symbol represents a single mice, with the bar indicating the mean. (e) Graph showing the absolute number of cells in BM and spleen at basal and at day 5 postadministration of $\mathrm{PHZ}$. (f) Representative flow cytometry of nucleated BM and spleen cells staining for CD71 and Ter119 differentiation markers and FSC distribution at day 5 postadministration of $\mathrm{PHZ}$. Percentage of cells in the individual sub-populations is indicated in each quadrant. (g) Graph showing the absolute number of Ter119+ and (h) ProE, Ery.A, Ery.B and Ery.C cells in BM and spleen at day 5 postadministration of PHZ. (i) Ery.A/Ery.B ratio in BM and spleen at day 5 postadministration of PHZ. Values represent the mean of at least eight mice of each genotype. *Statistically significant difference $(P<0.05)$ 
Nowadays, there is considerable excitement about the prospect of anticancer compounds that act through targeting PARP proteins, although non-isoform-selective PARP inhibitors are available. ${ }^{14}$ Our observations reveal potential offtarget effects that may arise from the use of such compounds. ${ }^{62}$ In addition, the specific role of PARP-2 in RS, a major driver of genomic instability, ${ }^{63-65}$ may have implications in the design and use of drugs targeting PARP proteins.

\section{Materials and Methods}

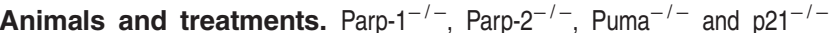
mice have been described previously. ${ }^{46,66-68} \mathrm{P} 21^{-I-}$ mice were bred with Parp $-1^{-1-}$ or Parp-2 $2^{-1-}$ mice, whereas Puma ${ }^{-1-}$ mice were bred with Parp- $2^{-1-}$ mice, to generate heterozygous mice, which were then bred to generate all genotypes. Genotyping was performed by PCR analysis using tail DNA as described. ${ }^{46,68-70}$ To induce acute anemia, mice were injected intraperitoneally with $\mathrm{PHZ}$ (Sigma-Aldrich, St. Louis, MO, USA) at $40 \mathrm{mg} / \mathrm{kg}$ on day 0,1 and 3. All mice were in C57BL6 background and were kept under pathogen-free conditions in the animal house facility at the Barcelona Biomedical Research Park (Barcelona, Spain). Animal studies were approved by the Institutional Animal Care and Use Committee.

Blood analysis. For cell analysis, peripheral blood from 10- to 12-week-old mice was collected in EDTA-coated vials. Blood cell parameters were measured immediately after sample collection using an Abacus Junior Vet Hematology Blood Analyzer (Diatron, Hialeah, FL, USA). Blood smears were air dried and stained with May-Grünwald Giemsa for routine analysis. Differential cell counts were made blind on more than 1000 cells on random areas of slides.

EPO levels in plasma were determined by ELISA Assay Kit (R\&D Systems, Minneapolis, MN, USA) in accordance with the manufacturer's instructions. Haptoglobin and hemoglobin levels in serum were determined by ELISA Assay Kits (Abcam, Cambridge, UK) in accordance with the manufacturer's instructions. Serum concentrations of total and direct bilirubin were determined by using a Bilirubin Assay Kit (BioAssay Systems, Hayward, CA, USA) in accordance with the manufacturer's instructions. Indirect bilirubin was determined by subtracting the concentration of direct bilirubin from the total bilirubin concentration. Iron levels in serum were measured on the Cobas 8000-701 Clinical Analyzer (Roche, Foster City, CA, USA).

Histological analysis. To study in situ splenic hemosiderin deposits, a Perl's iron stain technique was performed in $3 \mu \mathrm{m}$ sections of samples from spleen of wildtype and Parp-2-1- mice. The staining was performed by using an Automated Stain System (Dako Artisan, Dako, Glostrup, Denmark), following the manufacturer's recommendations (Dako Artisan Iron Stain Kit, Dako Artisan). Hemosiderin was identified as blue deposits within cells. For fetal liver histological examination, embryos were fixed in formalin and embedded in paraffin for subsequent processing. Consecutive $2.5-\mu \mathrm{m}$ sections were treated with citrate for antigen recovery and processed for immunohistochemistry with antibody against Ter119 (BD Biosciences, San Jose, CA, USA). Hematoxylin was used to counterstain Immunohistochemistry slides were scanned with a MIRAX digitalized system (Zeiss, Oberkochen, Germany).

Flow cytometry and cell sorting. Cell suspensions were washed in PBS, resuspended in PBS containing $0.5 \%$ BSA and incubated with antibodies on ice for $30 \mathrm{~min}$. Incubation with biotin-labeled antibodies was followed by incubation with streptavidin conjugates. Enucleated cells were lysed using ACK Lysis Buffer (BioWhitaker, Verviers, Belgium). Cell doublets were excluded from all analyses and dead cells were excluded by the use of DAPI. For intracellular staining, cells were stained for cell-surface markers, fixed and made permeable by using an intracellular staining buffer set (BD Biosciences), and then stained with specific antibody or isotype control. The following antibodies were used: PE-conjugated Ter119 (Ter119; BD Biosciences), APC-conjugated CD71 (R17217; eBiosciences, San Diego, CA, USA), phospho-H2AX (S139) (JPW301; Millipore, Billerica, MA, USA), phosphoCHK1 (S345) (133D3; Cell Signaling Technology, Danvers, MA, USA), phosphoRPA32 (S4/8) (Bethyl Laboratories, Montgomery, TX, USA), PerCP-conjugated streptavidin (BioLegend, San Diego, CA, USA) and goat Alexa Fluor 488- or 555conjugated (Invitrogen, Carlsbad, CA, USA) anti-rabbit and anti-mouse antibody.
Samples were acquired with an LSRII cytometer (BD Biosciences) and the data were analyzed with the DIVA (BD Biosciences) and FlowJo (TreeStar) software (FlowJo, Ashland, OR, USA). Cell sorting was performed in a FACS Aria II SORP (BD Biosciences).

Reticulocyte index. Reticulocytes in blood were determined by staining with thiazole orange and analyses by flow cytometry. The reticulocyte index was calculated as reticulocyte (\%)x(hematocrit (\%)/normal mouse hematocrit (\%)).

RBC turnover assay. RBCs were labeled in vivo with biotin by intravenous injection of mice with $60 \mathrm{mg} / \mathrm{kg}$ of NHS-LC-biotin (N-succinimidyl-6-(biotinamido) hexanoate) (Pierce Biotechnology, Rockford, IL, USA). The percentage of biotinylated cells in circulating blood was determined at regular intervals. For monitoring, blood samples were obtained from the tail vein, diluted in PBS, labeled with APC-conjugated streptavidin (BD Biosciences) and FITC-conjugated Ter119 and analzsed by flow cytometry.

Colony formation assays. Cell suspensions were mixed with MethoCult M3434 methyl cellulose semisolid medium (StemCell Technologies, Grenoble, France), plated in $35-\mathrm{mm}$ dishes and cultured at $37^{\circ} \mathrm{C}$ with $5 \% \mathrm{CO}_{2}$. CFU-E colonies were scored on day 3 , whereas BFU-E colonies were scored on day 7 .

Cell cycle analysis. For in vivo BrdU labeling experiments, 10- to 12-week-old mice received a single intraperitoneal injection of BrdU (BD Biosciences; $1 \mathrm{mg} / 6 \mathrm{~g}$ of mouse weight). Cells were isolated from mice $1 \mathrm{~h}$ after injection, surface stained for CD71 and Ter119, fixed, permeabilized and intracellularly stained with anti-BrdUFITC using the BrdU Flow Kit (BD Biosciences) and DAPI to gauge the amount of DNA per cell.

Immunofluorescence microscopy. Nucleated Ter $119^{+}$cells from BM were sorted on slides, fixed in $2 \%$ paraformaldehyde/PBS (w/v) for $10 \mathrm{~min}$ at room temperature, washed in PBS and permeabilized in a $0.1 \%$ sodium citrate $/ 0.1 \%$ Triton X-100 solution. Slides were then incubated in blocking solution, and incubated overnight with a rabbit polyclonal anti-active caspase-3 (R\&D Systems) primary antibody at $4{ }^{\circ} \mathrm{C}$. After washing, sections were incubated for $1 \mathrm{~h}$ at room temperature with goat anti-rabbit-conjugated Alexa Fluor 488, counterstained with DAPI, mounted and examined with an inverted fluorescence microscopy (Olympus BX61 microscope, Tokyo, Japan).

Western blot. Nucleated Ter $119^{+}$sorted cells from wild-type and Parp-2-1mice were washed once with PBS, and total protein extracts were prepared by directly adding $2 \times$ NuPAGE LDS sample buffer and then incubated for $5 \mathrm{~min}$ at $95^{\circ} \mathrm{C}$. Samples were resolved by SDS-PAGE and analyzed by standard western blotting techniques. Antibodies against CHK1 (2G1D5; Cell Signaling Technology), phospho-CHK1 (S345), $\beta$-actin (Sigma-Aldrich) and phospho-RPA32 (S4/8) were used. Signals were developed using an Enhanced Chemiluminiscence Kit (Amersham-Pharmacia Biotech, Buckinghamshire, UK), according to the manufacturer's instructions.

RNA extraction, reverse transcription and PCR. Total RNA was isolated from sorted nucleated Ter $119^{+}$cells from BM of wild-type and Parp-2 ${ }^{-1-}$ mice by using the RNeasy Total RNA Isolation Kit (Qiagen, Valencia, CA, USA) with on-column DNase I (Qiagen) digestion, following the manufacturer's instructions. Three independent experiments with different batches of cells were carried out. Quantity and integrity of the RNA was assessed by nanoelectrophoresis using the Pico Lab-on-a-Chip assay for total eukaryotic RNA using Bioanalyzer 2100 (Agilent Technologies, Palo Alto, CA, USA). Total RNA ( $5 \mathrm{ng}$ ) from each sample was used for cDNA synthesis using the Ovation Pico WTA System V2 (NuGEN Technologies, San Carlos, CA, USA). Specific primers for the different genes are included in Supplementary Table S2. qPCR was carried out using $15 \mathrm{ng}$ cDNA per sample using platinum SYBR Green Master mix (Applied Biosystems, Warrington, UK). Assays were run in triplicate on the ABI $7900 \mathrm{HT}$ system (Applied Biosystems). Expression levels of $\beta$-actin were used to normalize the amount of the examined transcript.

Gene expression array. Total RNA was isolated from sorted nucleated Ter $119^{+}$cells derived from pooled BM of three mice from each genotype. Microarray expression analysis was performed from samples of two independent experiments. Quantity and integrity of the RNA was assessed by nanoelectrophoresis using the 
Pico Lab-on-a-Chip assay for total eukaryotic RNA using Bioanalyzer 2100 (Agilent Technologies). Only samples with high integrity (RNA integrity number $>8$ ) were subsequently used in microarray experiments. Microarray expression profiles were obtained using the Affymetrix GeneChip Mouse Gene 1.0 ST Array (Affymetrix, Santa Clara, CA, USA) and the GCS3000 Affymetrix Platform (Affymetrix). Briefly, $10 \mathrm{ng}$ of total RNA from each sample was amplified using the Ovation Pico WTA System (NuGEN Technologies) and sense transcript cDNA (ST-cDNA) was generated using the WT-Ovation Exon Module (NuGEN Technologies). After, STcDNA was fragmented and labeled with the FL-Ovation cDNA Biotin Module V2 (NuGEN Technologies), and the biotinylated CDNA was hybridized to Affymetrix GeneChip Mouse Gene 1.0 ST Arrays. Following hybridization, the array was washed and stained, and finally scanned to generate CEL files for each array. Microarray data have been deposited into the Gene Expression Omnibus (GSE54864).

Statistical analysis. The log-rank test was used to determine the statistical of animal survival. All other statistical analyses used a $t$-test (two-tailed, assuming unequal variance). $P$-values of $<0.05$ were considered to indicate statistical significance. Bioinformatics statistical analysis of microarray data is indicated in the Supplementary Data.

\section{Conflict of Interest}

The authors declare no conflict of interest.

Acknowledgements. We thank $\mathrm{J}$ Moreno, $\mathrm{M}$ Juan and $\mathrm{S}$ Capdevila for assistance with mice, R Gimeno, A Bigas and C Gekas for useful comments, O Fornes for cell sorting and flow cytometry assistance, E Puigdecanet and L Nonell for microarrays support and S Mojal for statistical analysis support, as well as A Strasser for Puma-deficient mice. This work was supported by the Spanish Ministerio de Economía y Competitividad (SAF2011-26900), Generalitat de Catalunya (2014/SGR/184) and Fundació La Marató de TV3 (20134130) to JY. Work in FD and VS is supported by the Centre National de la Recherche Scientifique, Université de Strasbourg, Ligue contre le Cancer (Equipe labellisée) and Laboratory of Excellence (LabEx) Medalis ANR-10-LABX-0034 'Programme d'investissement d'avenir'. AV and EO are supported by the Austrian Science Fund (FWF; W1101, MCBO). Work in OF-C laboratory is supported by the Spanish Ministry of Science (SAF2011-23753), Association for International Cancer Research (12-0229), Fundació La Marató de TV3 (20134130) and European Research Council (ERC-210520). AJL-C is a recipient of a postdoctoral fellowship from the Spanish Association Against Cancer (AECC). LL was a recipient of a postdoctoral fellowship from the Juan de la Cierva Program.

\section{Author contributions}

JF and LL carried out most of the experiments presented in the paper. JM-C carried out RBC lifespan and blood cell analysis experiments. CM and AJL-C carried out pathology and microscopy experiments. $\mathrm{JL}$ performed gene expression array analysis. CA and EO provided technical assistance. JN and LF carried out blood cell analysis. FD, VS, AV and OF-C designed experiments, provided reagents and edited paper. JY planned and designed experiments, performed experiments and wrote the paper. All authors discussed the results and commented on the manuscript.

1. Hattangadi SM, Wong P, Zhang L, Flygare J, Lodish HF. From stem cell to red cell: regulation of erythropoiesis at multiple levels by multiple proteins, RNAs, and chromatin modifications. Blood 2011; 118: 6258-6268.

2. Palis J, Segel GB. Developmental biology of erythropoiesis. Blood Rev 1998; 12: 106-114.

3. Socolovsky M, Nam H, Fleming MD, Haase VH, Brugnara C, Lodish HF. Ineffective erythropoiesis in Stat5a(-I-)5b(-/-) mice due to decreased survival of early erythroblasts. Blood 2001; 98: 3261-3273.

4. Shearstone JR, Pop R, Bock C, Boyle P, Meissner A, Socolovsky M. Global DNA demethylation during mouse erythropoiesis in vivo. Science 2011; 334: 799-802.

5. Sankaran VG, Ludwig LS, Sicinska E, Xu J, Bauer DE, Eng JC et al. Cyclin D3 coordinates the cell cycle during differentiation to regulate erythrocyte size and number. Genes Dev 2012; 26: 2075-2087.

6. Wang F, Zhu Y, Guo L, Dong L, Liu H, Yin $\mathrm{H}$ et al. A regulatory circuit comprising GATA1/2 switch and microRNA-27a/24 promotes erythropoiesis. Nucleic Acids Res 2014; 42: $442-457$.

7. Grover A, Mancini E, Moore S, Mead AJ, Atkinson D, Rasmussen KD et al. Erythropoietin guides multipotent hematopoietic progenitor cells toward an erythroid fate. J Exp Med 2014; 211: 181-188.
8. Balarajan Y, Ramakrishnan U, Ozaltin E, Shankar AH, Subramanian SV. Anaemia in lowincome and middle-income countries. Lancet 2011; 378: 2123-2135.

9. Yelamos J, Farres J, Llacuna L, Ampurdanes C, Martin-Caballero J. PARP-1 and PARP-2: new players in tumour development. Am J Cancer Res 2011; 1: 328-346.

10. Jungmichel S, Rosenthal F, Altmeyer M, Lukas J, Hottiger MO, Nielsen ML. Proteome-wide identification of poly(ADP-Ribosyl)ation targets in different genotoxic stress responses. Mol Cell 2013; 52: 272-285.

11. Rulten SL, Fisher AE, Robert I, Zuma MC, Rouleau M, Ju L et al. PARP-3 and APLF function together to accelerate nonhomologous end-joining. Mol Cell 2011; 41: 33-45.

12. Boehler C, Gauthier LR, Mortusewicz O, Biard DS, Saliou JM, Bresson A et al. Poly(ADP-ribose) polymerase 3 (PARP3), a newcomer in cellular response to DNA damage and mitotic progression. Proc Natl Acad Sci USA 2011; 108: 2783-2788.

13. Lord CJ, Ashworth A. The DNA damage response and cancer therapy. Nature 2012; 481: 287-294.

14. Wahlberg E, Karlberg T, Kouznetsova E, Markova N, Macchiarulo A, Thorsell AG, Pol E, Frostell A, Ekblad T, Oncu D, Kull B, Robertson GM, Pellicciari R, Schuler H, Weigelt J. Family-wide chemical profiling and structural analysis of PARP and tankyrase inhibitors. Nat. Biotechnol. 2012; 30: 283-288.

15. Leger K, Bar D, Savic N, Santoro R, Hottiger MO. ARTD2 activity is stimulated by RNA. Nucleic Acids Res. 2014; 42: 5072-5082.

16. Isabelle M, Moreel X, Gagne JP, Rouleau M, Ethier C, Gagne P et al. Investigation of PARP-1, PARP-2, and PARG interactomes by affinity-purification mass spectrometry. Proteome Sci 2010; 8: 22.

17. Oliver AW, Ame JC, Roe SM, Good V, de Murcia G, Pearl LH. Crystal structure of the catalytic fragment of murine poly(ADP-ribose) polymerase-2. Nucleic Acids Res 2004; 32 : 456-464.

18. Troiani S, Lupi R, Perego R, Depaolini SR, Thieffine S, Bosotti R et al. Identification of candidate substrates for poly(ADP-ribose) polymerase-2 (PARP2) in the absence of DNA damage using high-density protein microarrays. FEBS J 2011; 278: 3676-3687.

19. Yelamos J, Monreal Y, Saenz L, Aguado E, Schreiber V, Mota R et al. PARP-2 deficiency affects the survival of $C D 4+C D 8+$ double-positive thymocytes. EMBO J 2006; 25 : 4350-4360.

20. Nicolas L, Martinez C, Baro C, Rodriguez M, Baroja-Mazo A, Sole F et al. Loss of poly(ADP-ribose) polymerase-2 leads to rapid development of spontaneous T-cell lymphomas in p53-deficient mice. Oncogene 2010; 29: 2877-2883.

21. Dantzer F, Mark M, Quenet D, Scherthan H, Huber A, Liebe B et al. Poly(ADP-ribose) polymerase-2 contributes to the fidelity of male meiosis I and spermiogenesis. Proc Natl Acad Sci USA 2006; 103: 14854-14859.

22. Robert I, Dantzer F, Reina-San-Martin B. Parp1 facilitates alternative NHEJ, whereas Parp2 suppresses $\mathrm{lgH} / \mathrm{c}$-myc translocations during immunoglobulin class switch recombination. J Exp Med 2009; 206: 1047-1056.

23. Bai P, Houten SM, Huber A, Schreiber V, Watanabe M, Kiss B et al. Poly(ADP-ribose) polymerase-2 [corrected] controls adipocyte differentiation and adipose tissue function through the regulation of the activity of the retinoid $\mathrm{X}$ receptor/peroxisome proliferatoractivated receptor-gamma [corrected] heterodimer. J Biol Chem 2007; 282: 37738-37746.

24. Bai P, Canto C, Brunyanszki A, Huber A, Szanto M, Cen Y et al. PARP-2 regulates SIRT1 expression and whole-body energy expenditure. Cell Metab 2011; 13: 450-460.

25. Szanto M, Brunyanszki A, Marton J, Vamosi G, Nagy L, Fodor T et al. Deletion of PARP-2 induces hepatic cholesterol accumulation and decrease in HDL levels. Biochim Biophys Acta 2014; 1842: 594-602.

26. Farres J, Martin-Caballero J, Martinez C, Lozano JJ, Llacuna L, Ampurdanes C et al. Parp-2 is required to maintain hematopoiesis following sublethal gamma-irradiation in mice. Blood 2013; 122: 44-54.

27. Paulson RF, Shi L, Wu DC. Stress erythropoiesis: new signals and new stress progenitor cells. Curr Opin Hematol 2011; 18: 139-145.

28. Van Putten $L M$. The life span of red cells in the rat and the mouse as determined by labeling with DFP32 in vivo. Blood 1958; 13: 789-794.

29. Connor J, Pak CC, Schroit AJ. Exposure of phosphatidylserine in the outer leaflet of human red blood cells. Relationship to cell density, cell age, and clearance by mononuclear cells. J Biol Chem 1994; 269: 2399-2404.

30. de JK, Emerson RK, Butler J, Bastacky J, Mohandas N, Kuypers FA. Short survival of phosphatidylserine-exposing red blood cells in murine sickle cell anemia. Blood 2001; 98: 1577-1584.

31. Chan KL, Palmai-Pallag T, Ying S, Hickson ID. Replication stress induces sister-chromatid bridging at fragile site loci in mitosis. Nat Cell Biol 2009; 11: 753-760.

32. Naim V, Rosselli F. The FANC pathway and mitosis: a replication legacy. Cell Cycle 2009; 8: 2907-2911.

33. Crasta K, Ganem NJ, Dagher R, Lantermann AB, Ivanova EV, Pan $\mathrm{Y}$ et al. DNA breaks and chromosome pulverization from errors in mitosis. Nature 2012; 482: 53-58.

34. Liu Y, Pop R, Sadegh C, Brugnara C, Haase VH, Socolovsky M. Suppression of Fas-FasL coexpression by erythropoietin mediates erythroblast expansion during the erythropoietic stress response in vivo. Blood 2006; 108: 123-133.

35. Fernandez-Capetillo O, Lee A, Nussenzweig M, Nussenzweig A. H2AX: the histone guardian of the genome. DNA Repair (Amst) 2004; 3: 959-967. 
36. Toledo LI, Murga M, Zur R, Soria R, Rodriguez A, Martinez S et al. A cell-based screen identifies ATR inhibitors with synthetic lethal properties for cancer-associated mutations. Nat Struct Mol Biol 2011; 18: 721-727.

37. Syljuasen RG, Sorensen CS, Hansen LT, Fugger K, Lundin C, Johansson F et al. Inhibition of human Chk1 causes increased initiation of DNA replication, phosphorylation of ATR targets, and DNA breakage. Mol Cell Biol 2011; 25: 3553-3562.

38. Lopez-Contreras AJ, Fernandez-Capetillo 0 . The ATR barrier to replication-born DNA damage. DNA Repair (Amst) 2010; 9: 1249-1255.

39. Ward IM, Minn K, Chen J. UV-induced ataxia-telangiectasia-mutated and Rad3-related (ATR) activation requires replication stress. J Biol Chem 2004; 279: 9677-9680.

40. Liu S, Opiyo SO, Manthey K, Glanzer JG, Ashley AK, Amerin C et al. Distinct roles for DNAPK, ATM and ATR in RPA phosphorylation and checkpoint activation in response to replication stress. Nucleic Acids Res 2012; 40: 10780-10794.

41. Subramanian A, Tamayo P, Mootha VK, Mukherjee S, Ebert BL, Gillette MA et al. Gene set enrichment analysis: a knowledge-based approach for interpreting genome-wide expression profiles. Proc Natl Acad Sci USA 2005; 102: 15545-15550.

42. Socolovsky M, Fallon AE, Wang S, Brugnara C, Lodish HF. Fetal anemia and apoptosis of red cell progenitors in Stat5a $-/-5 b-/$ - mice: a direct role for Stat5 in Bcl-X(L) induction. Cell 1999; 98: 181-191.

43. Itano HA, Hirota K, Hosokawa K. Mechanism of induction of haemolytic anaemia by phenylhydrazine. Nature 1975; 256: 665-667.

44. Schlegel R, MacGregor JT, Everson RB. Assessment of cytogenetic damage by quantitation of micronuclei in human peripheral blood erythrocytes. Cancer Res 1986; 46: 3717-3721.

45. Heddle JA, Hite M, Kirkhart B, Mavournin K, MacGregor JT, Newell GW et al. The induction of micronuclei as a measure of genotoxicity. A report of the US Environmental Protection Agency Gene-Tox Program. Mutat Res 1983; 123: 61-118.

46. Menissier de Murcia J, Ricoul M, Tartier L, Niedergang C, Huber A, Dantzer F et al. Functional interaction between PARP-1 and PARP-2 in chromosome stability and embryonic development in mouse. EMBO J 2003; 22: 2255-2263.

47. Saxena A, Wong LH, Kalitsis P, Earle E, Shaffer LG, Choo KH. Poly(ADP-ribose) polymerase 2 localizes to mammalian active centromeres and interacts with PARP-1, Cenpa, Cenpb and Bub3, but not Cenpc. Hum Mol Genet 2002; 11: 2319-2329.

48. Rogakou EP, Pilch DR, Orr AH, Ivanova VS, Bonner WM. DNA double-stranded breaks induce histone H2AX phosphorylation on serine 139. J Biol Chem 1998; 273: 5858-5868.

49. Ward IM, Chen J. Histone H2AX is phosphorylated in an ATR-dependent manner in response to replicational stress. J Biol Chem 2001; 276: 47759-47762.

50. Furuta T, Takemura H, Liao ZY, Aune GJ, Redon C, Sedelnikova OA et al. Phosphorylation of histone $\mathrm{H} 2 \mathrm{AX}$ and activation of Mre11, Rad50, and Nbs1 in response to replicationdependent DNA double-strand breaks induced by mammalian DNA topoisomerase cleavage complexes. J Biol Chem 2003; 278: 20303-20312.

51. Ewald B, Sampath D, Plunkett W. H2AX phosphorylation marks gemcitabine-induced stalled replication forks and their collapse upon S-phase checkpoint abrogation. Mol Cancer Ther 2007; 6: 1239-1248

52. Gagou ME, Zuazua-Villar P, Meuth M. Enhanced H2AX phosphorylation, DNA replication fork arrest, and cell death in the absence of Chk1. Mol Biol Cell 2010; 21: 739-752.

53. Szanto M, Brunyanszki A, Kiss B, Nagy L, Gergely P, Virag L et al. Poly(ADP-ribose) polymerase-2: emerging transcriptional roles of a DNA-repair protein. Cell Mol Life Sci 2012 69: 4079-4092.
54. Porlan E, Morante-Redolat JM, Marques-Torrejon MA, Andreu-Agullo C, Carneiro C, Gomez-lbarlucea $\mathrm{E}$ et al. Transcriptional repression of Bmp2 by p21(Waf1/Cip1) links quiescence to neural stem cell maintenance. Nat Neurosci 2013; 16: 1567-1575.

55. Viale A, De FF, Orleth A, Cambiaghi V, Giuliani V, Bossi D et al. Cell-cycle restriction limits DNA damage and maintains self-renewal of leukaemia stem cells. Nature 2009; 457: 51-56.

56. Murga M, Bunting S, Montana MF, Soria R, Mulero F, Canamero M et al. A mouse model of ATR-Seckel shows embryonic replicative stress and accelerated aging. Nat Genet 2009; 41: 891-898.

57. Sugimura K, Takebayashi S, Taguchi H, Takeda S, Okumura K. PARP-1 ensures regulation of replication fork progression by homologous recombination on damaged DNA. J Cell Biol 2008; 183: 1203-1212.

58. Ray CA, Hashimoto $\mathrm{Y}$, Herrador R, Neelsen KJ, Fachinetti D, Bermejo R et al. Topoisomerase I poisoning results in PARP-mediated replication fork reversal. Nat Struct Mol Biol 2012; 19: 417-423.

59. McLellan JL, O'Neil NJ, Barrett I, Ferree E, van Pel DM, Ushey K et al. Synthetic lethality of cohesins with PARPs and replication fork mediators. PLoS Genet 2012; 8: e1002574.

60. Min W, Bruhn C, Grigaravicius P, Zhou ZW, Li F, Kruger A et al. Poly(ADP-ribose) binding to Chk1 at stalled replication forks is required for S-phase checkpoint activation. Nat Commun 2013; 4: 2993

61. Bryant HE, Petermann E, Schultz N, Jemth AS, Loseva O, Issaeva $\mathrm{N}$ et al. PARP is activated at stalled forks to mediate Mre11-dependent replication restart and recombination. EMBO J 2009; 28: 2601-2615.

62. Fong PC, Boss DS, Yap TA, Tutt A, Wu P, Mergui-Roelvink M et al. Inhibition of poly(ADPribose) polymerase in tumors from BRCA mutation carriers. N Engl J Med 2009; 361 : 123-134.

63. Burrell RA, McClelland SE, Endesfelder D, Groth P, Weller MC, Shaikh N et al. Replication stress links structural and numerical cancer chromosomal instability. Nature 2013; 494: 492-496.

64. Hu L, Filippakis H, Huang H, Yen TJ, Gjoerup OV. Replication stress and mitotic dysfunction in cells expressing simian virus 40 large T antigen. J Virol 2013; 87: 13179-13192.

65. Ichijima Y, Yoshioka K, Yoshioka Y, Shinohe K, Fujimori H, Unno J et al. DNA lesions induced by replication stress trigger mitotic aberration and tetraploidy development. PLoS One 2010; 5: e8821.

66. de Murcia JM, Niedergang C, Trucco C, Ricoul M, Dutrillaux B, Mark M et al. Requirement of poly(ADP-ribose) polymerase in recovery from DNA damage in mice and in cells. Proc Natl Acad Sci USA 1997; 94: 7303-7307.

67. Brugarolas J, Chandrasekaran C, Gordon JI, Beach D, Jacks T, Hannon GJ et al. Radiationinduced cell cycle arrest compromised by p21 deficiency. Nature 1995; 377: 552-557.

68. Villunger A, Michalak EM, Coultas L, Mullauer F, Bock G, Ausserlechner MJ. p53- and druginduced apoptotic responses mediated by BH3-only proteins puma and noxa. Science 2003; 302: 1036-1038.

69. Corral J, Yelamos J, Hernandez-Espinosa D, Monreal Y, Mota R, Arcas I et al. Role of lipopolysaccharide and cecal ligation and puncture on blood coagulation and inflammation in sensitive and resistant mice models. Am J Pathol 2005; 166: 1089-1098.

70. Garcia-Fernandez RA, Garcia-Palencia P, Sanchez MA, Gil-Gomez G, Sanchez B, Rollan E et al. Combined loss of p21(waf1/cip1) and p27(kip1) enhances tumorigenesis in mice. Lab Invest 2011: 91: 1634-1642.

Supplementary Information accompanies this paper on Cell Death and Differentiation website (http://www.nature.com/cdd) 